\begin{tabular}{|c|l|}
\hline Title & Stereoselective synthesis of a C1-C6 fragment of pinnatoxin A via a 1,4 addition/Alkylation sequence \\
\hline Author(s) & Nakamura, Seiichi; Kikuchi, Fumiaki; Hashimoto, Shunichi \\
\hline Citation & $\begin{array}{l}\text { Tetrahedron A symmetry, 19(9), 1059-1067 } \\
\text { https://doi.org/10.1016/.tetasy.2008.04.013 }\end{array}$ \\
\hline Issue Date & 2008-05-16 \\
\hline Doc URL & http://hdl.handle.net/2115/33049 \\
\hline Type & article (author version) \\
\hline File Information & TETASY 57110.pdf \\
\hline
\end{tabular}

Instructions for use 


\title{
Stereoselective synthesis of a C1-C6 fragment of pinnatoxin A via a 1,4-addition/alkylation sequence
}

\author{
Seiichi Nakamura, Fumiaki Kikuchi and Shunichi Hashimoto* \\ Faculty of Pharmaceutical Sciences, Hokkaido University, Sapporo 060-0812, Japan
}

\begin{abstract}
A C1-C6 fragment of pinnatoxin A, (5S,6R)-5,6-dimethyl-3-methyleneoxepan-2-one, which features a $\gamma, \delta$-trans-dimethylsubstituted $\alpha$-methylene lactone, has been synthesized in an optically pure form from ethyl $(E)$-4-benzyloxy-2-butenoate through an auxiliary-based conjugate addition and alkylation reaction. The excellent diastereoselectivity $(98: 2)$ observed in the alkylation reaction would be a result of stereocontrol from both the adjacent stereocenter and the chiral oxazolidinone. (C) 2008 Elsevier Science. All rights reserved
\end{abstract}

\section{Introduction}

Pinnatoxins ${ }^{1}$ and pteriatoxins, ${ }^{2}$ isolated and characterized by Uemura and co-workers in 1995 and 2001, respectively, are members of a class of marine toxins that are implicated in shellfish poisoning. ${ }^{3}$ Structurally, these molecules feature a 27-membered carbocyclic ring that comprises a $[6,5,6]$-dispiroketal (BCD ring), a [5,6]-bicycloketal (EF ring), and a [6,7]-spiroimine (AG ring). Not surprisingly, the unprecedented molecular architecture and biological activity, in conjunction with the scarcity of the natural material, have provided an impetus to develop a route to this class of shellfish toxins. ${ }^{4}$ The first total synthesis of (-)-pinnatoxin A was reported by Kishi and co-workers in 1998, leading to the absolute configurational assignment for the natural product. ${ }^{5}$ They also established the complete stereochemistry of pinnatoxins $\mathrm{B}$ and $\mathrm{C}^{6}$ and of pteriatoxins $\mathrm{A}-\mathrm{C}^{7}$ by total syntheses. A formal total synthesis of pinnatoxin A was documented by Hirama and co-workers, ${ }^{8}$ and synthetic approaches have been pursued extensively in the laboratories of Ishihara and Murai, ${ }^{9}$ Kitching ${ }^{10}$ and Zakarian. $^{11}$

As part of our own synthetic studies on these natural products, we have already reported a stereoselective construction of the $[6,5,6]$-dispiroketal ring system by exploiting a tandem hemiketal formation/hetero-Michael reaction sequence through the agency of LiOMe as a base. ${ }^{12}$ To construct the $\mathrm{G}$ ring portion of these molecules, a biomimetic intramolecular Diels-Alder reaction appeared attractive due to the concurrent formation of the 27-membered carbocycle.
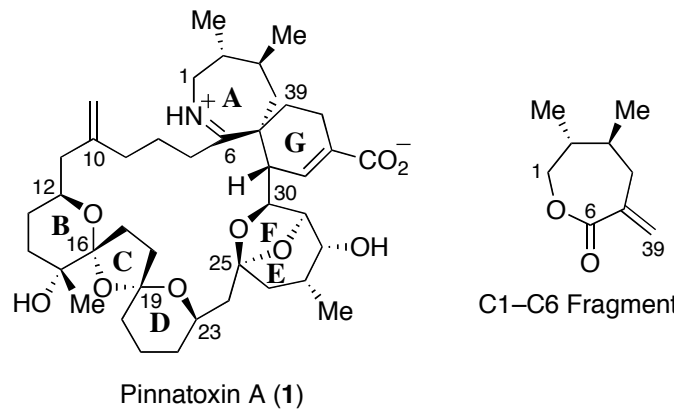

C1-C6 Fragment (2)

Indeed, Kishi and co-workers accomplished total syntheses by an intramolecular cycloaddition-based approach.,7a On the other hand, an intermolecular Diels-Alder approach has an advantage of allowing convenient access to simpler analogues for biological evaluation. The stereochemical arrangement of the $\mathrm{G}$ ring of pinnatoxins/pteriatoxins would require an exo-selective Diels-Alder reaction. In this regard, Roush and co-workers demonstrated the striking preference of conformationally $s$-cis-restricted enone and enoate dienophiles for exo cycloaddition. ${ }^{13,14}$ Based on these precedents, we elected to take advantage of an intermolecular reaction using $\alpha$-methylene lactone $\mathbf{2}$ as the dienophile for the synthesis of $\mathbf{1}$ and its congeners. Therefore, a practical route to optically pure lactone $\mathbf{2}$ needed to be developed. In this paper, we describe a stereoselective route to the lactone $\mathbf{2}$, in which a chiral auxiliary, $(R)-4$ phenyl-2-oxazolidinone, plays a pivotal role as the source of asymmetry for the creation of both stereocenters at $\mathrm{C} 2$ and $\mathrm{C} 3$.

\footnotetext{
* Corresponding author. Tel.: +81-11-706-3236; fax: +81-11-706-4981; email: hsmt@pharm.hokudai.ac.jp
}

\section{Results and discussion}


The C1-C6 fragment 2 features a 7-membered $\alpha-$ methylene lactone containing branched methyl groups with a 1,2-trans relationship. To construct the methyl-bearing stereocenters at $\mathrm{C} 2$ and $\mathrm{C} 3$, we planned to take advantage of asymmetric reactions using a common chiral oxazolidinone: 1,4 -addition ${ }^{15}$ of a methylmagnesium reagent to a chiral $\alpha, \beta$-unsaturated 3-acyl-2-oxazolidinone 3 and subsequent alkylation with MeI (Scheme 1). However, to the

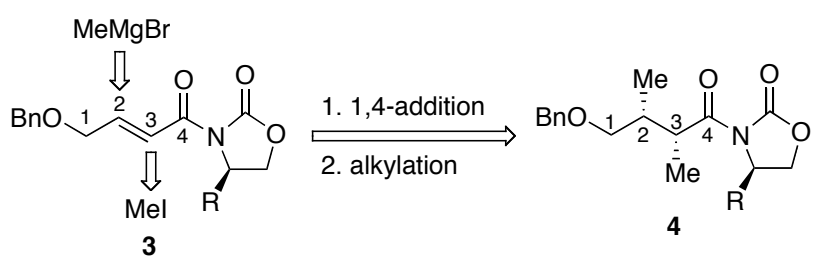<smiles>C=CC=CC(=O)OCCCC(=O)O</smiles>

Scheme 1. Plan for the synthesis of C1-C6 fragment 2.

best of our knowledge, the chiral oxazolidinone-based 1,4addition/alkylation sequence has not been employed to install 1,2-dimethyl arrangements along acyclic backbones. ${ }^{16}$ With regard to the oxazolidinone-based 1,4-addition reaction, Hruby and co-workers demonstrated that a phenyl-substituted auxiliary $(\mathrm{R}=\mathrm{Ph})$ is effective for asymmetric induction at the $\beta$-carbon. The major concern with this 1,4-addition/alkylation sequence was control of the configuration at the $\alpha$-carbon (C3) by alkylation, since Evans and co-workers demonstrated that the phenyl group is a less effective substituent to shield the diastereoface of the lithium enolate in alkylations of $\mathrm{N}$-butyryl-2-oxazolidinones with $\mathrm{MeI}$ at $-30{ }^{\circ} \mathrm{C}$ than any other groups examined, providing a 4.24:1 mixture of alkylation products. ${ }^{17}$

The synthesis of lactone 2 was initiated by $N$-acylation of the D-phenylglycine-derived oxazolidinone $\mathbf{8}^{15}$ with the known 4-benzyloxy-2-butenoic acid (7), ${ }^{18}$ obtained by hydrolysis of ethyl ester $6^{19}$ with $\mathrm{LiOH}$ in aqueous THF, under Merck conditions $\left[\mathrm{PivCl}, \mathrm{Et}_{3} \mathrm{~N}, \mathrm{LiCl}, \mathrm{THF}^{20}\right.$ to afford carboximide $9, \mathrm{mp} 115.5-116.5^{\circ} \mathrm{C},[\alpha]_{\mathrm{D}}{ }^{26}=-67.3$ (c 2.07, $\mathrm{CHCl}_{3}$ ), in $88 \%$ yield in two steps (Scheme 2). Conjugate addition of $\mathrm{MeMgBr}$ to the $\alpha, \beta$-unsaturated imide 9 was carried out according to Hruby conditions ${ }^{15}$ in the presence of $\mathrm{CuBr} \cdot \mathrm{SMe}_{2}$ in $\mathrm{THF} / \mathrm{Me}_{2} \mathrm{~S} / \mathrm{CH}_{2} \mathrm{Cl}_{2}(-78 \rightarrow$ $-30{ }^{\circ} \mathrm{C}$ ) to produce a mixture of 1,4 -adducts, $\mathbf{1 0}$ and its $\mathrm{C} 2$ epimer 11, in $97 \%$ yield in a ratio of $94: 6$ as determined by $500 \mathrm{MHz}{ }^{1} \mathrm{H} \mathrm{NMR}^{21}$ The minor diastereomer 11 could be removed by recrystallizations from $4: 1 \mathrm{AcOEt} / n$-hexane, providing diastereomerically pure carboximide 10, $\mathrm{mp}$ 68.5-69.0 ${ }^{\circ} \mathrm{C},[\alpha]_{\mathrm{D}}{ }^{22}=-52.8\left(c 2.08, \mathrm{CHCl}_{3}\right)$, as colorless needles. Our effort was then directed toward the crucial methylation reaction. Gratifyingly, the reaction of the sodium enolate generated from $\mathbf{1 0}$ with MeI in THF at $-78{ }^{\circ} \mathrm{C}$ exhibited excellent diastereoselectivity (98:2) for
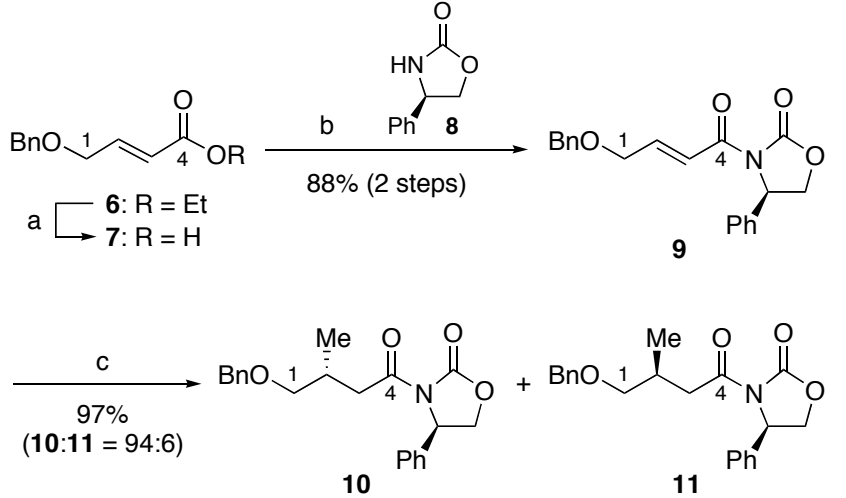

$d \downarrow d r=98: 2$

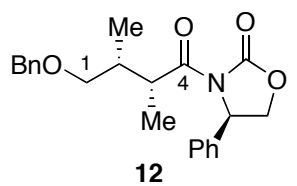

$83 \%$

Scheme 2. Reagents and conditions: (a) $\mathrm{LiOH}, 5: 1 \mathrm{THF} / \mathrm{H}_{2} \mathrm{O}, 72 \mathrm{~h}$; (b) $\mathrm{PivCl}, \mathrm{Et}_{3} \mathrm{~N}, \mathrm{THF},-20{ }^{\circ} \mathrm{C}, 2 \mathrm{~h}$, then oxazolidinone 8, LiCl, rt, $3 \mathrm{~h}$; (c) $\mathrm{MeMgBr}, \mathrm{CuBr} \cdot \mathrm{SMe}_{2}, 24: 8: 1 \mathrm{THF} / \mathrm{Me}_{2} \mathrm{~S} / \mathrm{CH}_{2} \mathrm{Cl}_{2},-78{ }^{\circ} \mathrm{C}, 30 \mathrm{~min}$, then $-30{ }^{\circ} \mathrm{C}, 30 \mathrm{~min}$; (d) NaHMDS, THF, $-78^{\circ} \mathrm{C}, 30 \mathrm{~min}$, then MeI, $6 \mathrm{~h}$.

the desired alkylation product 12 with 1,2-syn-dimethyl stereocenters. ${ }^{23}$ The excellent diastereoselection observed with $\mathbf{1 0}$ is attributed to the complementary influence on the facial bias imposed on the sodium enolate by the $\beta$-stereocenter as well as the chiral auxiliary. ${ }^{24}$ In this context, McGarvey and Williams reported that a good level of 1,2asymmetric induction from the $\beta$-substituent was observed in the methylation of ester enolates bearing oxygen substitution at a $\gamma$-position. ${ }^{25}$ For example, treatment of the lithium enolate derived from ester 13 with MeI in THF at $-78{ }^{\circ} \mathrm{C}$ afforded an 89:11 mixture of products favoring the syn-dimethyl-substituted ester $\mathbf{1 5}$ in $85 \%$ combined yield (Eq. 1). They reasoned that the homoallylic oxygen in the

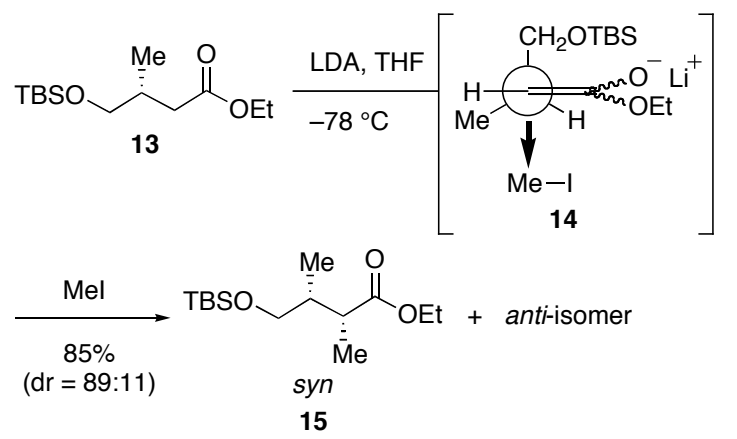

enolate would enhance the donor ability of the substituted carbon via lone pair participation, leading to the transition state 14, wherein the electron-donating $\mathrm{CH}_{2} \mathrm{OTBS}$ group is aligned anti to the forming bond and the sterically unencumbered hydrogen atom occupies the inside position. Based on the McGarvey precedent, the predominant 
formation of the 1,2-syn diastereomer 12 can be rationalized by the transition state $\mathbf{A}$, wherein the benzyloxymethyl group at $\mathrm{C} 2$ and the phenyl group of the auxiliary act in concert to direct the alkylation from the re-face of the enolate (Figure 1).

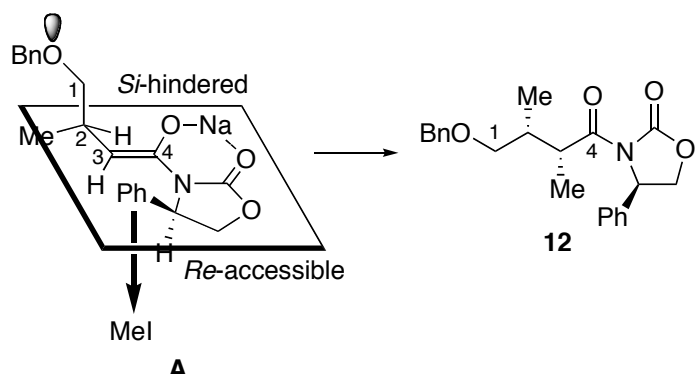

Figure 1. Stereochemical model for the predominant formation of synisomer 12 .

After chromatographic separation of the desired carboximide $12,[\alpha]_{\mathrm{D}}{ }^{20}=-82.0\left(c 2.01, \mathrm{CHCl}_{3}\right)$, reductive removal of the chiral auxiliary with $\mathrm{LiBH}_{4}$ in aqueous $\mathrm{THF}^{26}$ afforded the primary alcohol 16, $[\alpha]_{\mathrm{D}}{ }^{21}=+6.19$ (c 2.00, $\mathrm{CHCl}_{3}$ ), in $73 \%$ yield along with $66 \%$ recovery of the auxiliary (Scheme 3). Oxidation of alcohol 16 with DessMartin periodinane ${ }^{27}$ in $\mathrm{CH}_{2} \mathrm{Cl}_{2}$ was followed by HornerWadsworth-Emmons olefination with triethyl phosphonoacetate to give enoate $\mathbf{1 8}$ as an 8:1 $E / Z$ mixture of olefin isomers in $93 \%$ yield in two steps. Hydrogenation of the C4-C5 olefin and hydrogenolytic cleavage of the $\mathrm{C} 1$ benzyl protecting group were effected in a single operation by treatment of $\mathbf{1 8}$ with $\mathrm{Pd}(\mathrm{OH})_{2} / \mathrm{C}$ in AcOEt under a hydrogen atmosphere to afford $\varepsilon$-hydroxyester $19,[\alpha]_{\mathrm{D}}{ }^{22}=$ -10.6 (c 2.40, $\mathrm{CHCl}_{3}$ ), in 97\% yield. Saponification of 19 with $\mathrm{KOH}$ in $\mathrm{MeOH}$ and subsequent TsOH-catalyzed lactonization at high dilution (14 $\mathrm{mM}$ in benzene) provided 7-membered lactone 20, $[\alpha]_{\mathrm{D}}{ }^{21}=-43.1\left(c 2.02, \mathrm{CHCl}_{3}\right)$, in $90 \%$ yield in two steps.

With the 7-membered lactone $\mathbf{2 0}$ in hand, the remaining operation necessary for the synthesis of the C1-C6 fragment $\mathbf{2}$ was the $\alpha$-methylidenation of lactone $\mathbf{2 0}$. In this regard, Takeda and co-workers successfully utilized the Paterson procedure ${ }^{28}$ for the conversion of $\varepsilon$-caprolactone to $\alpha$-methylene- $\varepsilon$-caprolactone. ${ }^{14 \mathrm{~b}}$ Following this literature precedent, lactone $\mathbf{2 0}$ was uneventfully converted to the corresponding silyl ketene acetal, which, upon treatment with $\alpha$-chlorothioanisole in the presence of a catalytic amount of $\mathrm{ZnBr}_{2}$ in $\mathrm{CH}_{2} \mathrm{Cl}_{2}$, afforded $\alpha$-(phenylthiomethyl)lactone 21 as a 1:1 mixture of diastereomers in $58 \%$ yield in two steps along with $13 \%$ recovery of the lactone 20. Finally, oxidation of sulfide 21 with $\mathrm{NaIO}_{4}$ in aqueous $\mathrm{MeOH}$ and subsequent elimination of the corresponding sulfoxide in refluxing toluene furnished the target $\alpha$ methylene lactone $2,[\alpha]_{\mathrm{D}}{ }^{21}=-6.69\left(c 2.01, \mathrm{CHCl}_{3}\right)$, in $75 \%$ yield in two steps.
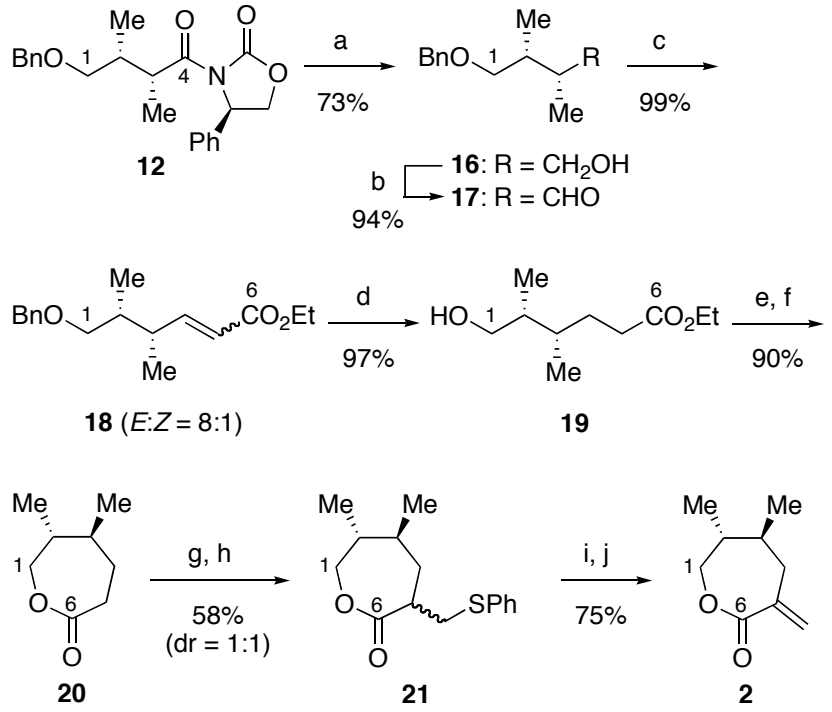

Scheme 3. Reagents and conditions: (a) $\mathrm{LiBH}_{4}, \mathrm{H}_{2} \mathrm{O}$, THF, $0{ }^{\circ} \mathrm{C}, 3 \mathrm{~h}$, then rt, $1.5 \mathrm{~h}$; (b) Dess-Martin periodinane, $\mathrm{CH}_{2} \mathrm{Cl}_{2}, 10 \mathrm{~min} ;$ (c) $(\mathrm{EtO})_{2} \mathrm{P}(\mathrm{O}) \mathrm{CH}_{2} \mathrm{CO}_{2} \mathrm{Et},{ }^{t} \mathrm{BuOK}, \mathrm{THF},-78{ }^{\circ} \mathrm{C}, 30 \mathrm{~min}$, then $0{ }^{\circ} \mathrm{C}, 30 \mathrm{~min}$; (d) $\mathrm{H}_{2}, 20 \% \mathrm{Pd}(\mathrm{OH})_{2} / \mathrm{C}$, AcOEt, $5 \mathrm{~h}$; (e) $\mathrm{KOH}, \mathrm{MeOH}, 4 \mathrm{~h}$; (f) $\mathrm{TsOH}$, benzene $(14 \mathrm{mM})$, reflux, $1 \mathrm{~h}$; $(\mathrm{g}) \mathrm{LDA}, \mathrm{THF},-78{ }^{\circ} \mathrm{C}, 1 \mathrm{~h}$, then $\mathrm{TMSCl}$, $-78{ }^{\circ} \mathrm{C}, 30 \mathrm{~min}$ and rt, $1 \mathrm{~h}$; (h) $\mathrm{PhSCH}_{2} \mathrm{Cl}, \mathrm{ZnBr}_{2}, \mathrm{CH}_{2} \mathrm{Cl}_{2}, 12 \mathrm{~h}$; (i) $\mathrm{NaIO}_{4}$, 9:1 $\mathrm{MeOH} / \mathrm{H}_{2} \mathrm{O}, 24 \mathrm{~h}$; (j) toluene, reflux, $3 \mathrm{~h}$.

\section{Conclusion}

We have achieved the stereoselective synthesis of a $\mathrm{C} 1-\mathrm{C} 6$ fragment of pinnatoxin A in 14 steps with an overall yield of $13.7 \%$. The foregoing synthetic sequence demonstrated the utility of phenyl-substituted oxazolidinone as a common chiral auxiliary to set the contiguous methylbearing stereocenters in a syn stereochemical relationship through a 1,4-addition of $\mathrm{MeMgBr}$ and subsequent alkylation with MeI wherein the facial bias conferred on the enolate was reinforced by the adjacent stereocenter created by the 1,4-addition. This is the first example of a stereoselective construction of a 1,2-syn-dimethyl array via the chiral oxazolidinone-based 1,4-addition/alkylation sequence. Efforts to complete the total synthesis of pinnatoxin A (1) using an intermolecular Diels-Alder strategy are in progress in our laboratory and will be reported in due course. ${ }^{29}$

\section{Experimental}

\subsection{General}

Melting points were determined on a Büchi 535 digital melting point apparatus and were uncorrected. Optical rotations were measured on a JASCO P-1030 digital polarimeter with a sodium lamp. Infrared (IR) spectra were recorded on JASCO FT/IR-5300 or FT/IR-4100 spectrometers and absorbance bands are reported in wavenumbers $\left(\mathrm{cm}^{-1}\right) .{ }^{1} \mathrm{H}$ NMR spectra were recorded on JEOL JNMECA500 (500 MHz) or Bruker ARX500 (500 MHz) spectrometers with tetramethylsilane $(\delta 0.00)$ or $\mathrm{C}_{6} \mathrm{H}_{6}(\delta$ 
7.20) as an internal standard. Coupling constants $(J)$ are reported in hertz $(\mathrm{Hz})$. Abbreviations of multiplicity are as follows: $\mathrm{s}$, singlet; $\mathrm{d}$, doublet; $\mathrm{t}$, triplet; $\mathrm{q}$, quartet; $\mathrm{m}$, multiplet; br, broad. Data are presented as follows: chemical shift, multiplicity, coupling constants, integration and assignment. Pinnatoxin numbering is used for proton assignments of all compounds. ${ }^{13} \mathrm{C}$ NMR spectra were recorded on JEOL JNM-EX270 (67.8 MHz), JNM-AL400 (100.6 MHz) or JNM-ECX400P (99.6 MHz) spectrometers with $\mathrm{CDCl}_{3}(\delta 77.0)$ as an internal standard. Electron impact (EI) and fast atom bombardment (FAB) mass spectra were recorded on JEOL JMS-FABmate and JMSHX110 spectrometers, respectively, by the Center for Instrumental Analysis, Hokkaido University.

Column chromatography was carried out on Kanto silica gel $60 \mathrm{~N}(63-210 \mu \mathrm{m})$. Analytical TLC was carried out on Merck Kieselgel $60 \quad \mathrm{~F}_{254}$ plates. Visualization was accomplished with ultraviolet light and anisaldehyde or phosphomolybdic acid stain, followed by heating.

Reagents and solvents were purified by standard means or used as received unless otherwise noted. Dehydrated stabilizer free THF was purchased from Kanto Chemical Co., Inc. Dichloromethane $\left(\mathrm{CH}_{2} \mathrm{Cl}_{2}\right)$ was distilled from $\mathrm{P}_{2} \mathrm{O}_{5}$ and redistilled from calcium hydride prior to use. Diisopropylamine $\left({ }^{i} \mathrm{Pr}_{2} \mathrm{NH}\right)$ and $\mathrm{TMSCl}$ were distilled from calcium hydride prior to use.

$\mathrm{CuBr} \cdot \mathrm{SMe}_{2}{ }^{30}$ and Dess-Martin periodinane ${ }^{31}$ were prepared according to literature procedures.

\section{2. $[4 R, 3(2 E)]-3-(4-B e n z y l o x y-2-b u t e n o y l)-4-p h e n y l-2-$} oxazolidinone (9). Lithium hydroxide monohydrate (4.67 g, $111 \mathrm{mmol}$ ) was added to a solution of ethyl 4-benzyloxy2-butenoate ${ }^{19}(6,12.3 \mathrm{~g}, 55.6 \mathrm{mmol})$ in 5:1 THF/ $\mathrm{H}_{2} \mathrm{O}(240$ $\mathrm{mL}$ ). After stirring for $72 \mathrm{~h}$, THF was removed in vacuo, and the residue was partitioned between $\mathrm{Et}_{2} \mathrm{O}(100 \mathrm{~mL})$ and $2 \mathrm{M}$ aqueous $\mathrm{NaHSO}_{4}(150 \mathrm{~mL})$. The aqueous layer was extracted with AcOEt $(300 \mathrm{~mL})$. The combined organic extracts were washed with brine $(100 \mathrm{~mL})$ and dried over anhydrous $\mathrm{Na}_{2} \mathrm{SO}_{4}$. Filtration and evaporation in vacuo furnished the crude product (11.0 g, slightly yellow solid), which was used without further purification.

Trimethylacetyl chloride $(9.3 \mathrm{~mL}, 61.2 \mathrm{mmol})$ was added to a solution of the crude carboxylic acid $7(11.0 \mathrm{~g})$ and $\mathrm{Et}_{3} \mathrm{~N}(24 \mathrm{~mL}, 167 \mathrm{mmol})$ in THF $(200 \mathrm{~mL})$ at $-25^{\circ} \mathrm{C}$ under an argon atmosphere. After stirring at $-20^{\circ} \mathrm{C}$ for $2 \mathrm{~h}, \mathrm{LiCl}$ $(3.5 \mathrm{~g}, 83.5 \mathrm{mmol})$ and 2-oxazolidinone $8^{15}$ (10.0 g, 61.2 $\mathrm{mmol}$ ) were added, and the mixture was allowed to warm to room temperature. After stirring for $3 \mathrm{~h}$, the reaction mixture was poured into an ice-cooled, two-layer mixture of $\mathrm{Et}_{2} \mathrm{O}(100 \mathrm{~mL})$ and saturated aqueous $\mathrm{NaHCO}_{3}(150$ $\mathrm{mL}$ ), and the whole mixture was extracted with AcOEt $(300 \mathrm{~mL})$. The organic extract was successively washed with saturated aqueous $\mathrm{NaHCO}_{3}(150 \mathrm{~mL})$ and brine $(2 \times 100 \mathrm{~mL})$, and dried over anhydrous $\mathrm{Na}_{2} \mathrm{SO}_{4}$. Filtration and evaporation in vacuo furnished the crude product $(21.7$ g, brown solid), which was recrystallized from 1:1 nhexane/AcOEt to give carboximide $9(13.9 \mathrm{~g}, 74 \%)$ as colorless plates. Concentration of the mother liquor followed by purified by column chromatography (silica gel $110 \mathrm{~g}, 3: 1 n$-hexane/AcOEt) gave $9(2.56 \mathrm{~g}, 14 \%)$ as a white solid: TLC $R_{f}=0.24$ (3:1 $n$-hexane/AcOEt); mp $115.5-116.5{ }^{\circ} \mathrm{C} ;[\alpha]_{\mathrm{D}}{ }^{26}=-67.3\left(c 2.07, \mathrm{CHCl}_{3}\right)$; IR (nujol) 2924, 2855, 1796, 1767, 1682, 1644, 1462, 1379, 1366, 1333, 1209, 1138, 972, $718 \mathrm{~cm}^{-1}$; ${ }^{1} \mathrm{H}$ NMR $(500 \mathrm{MHz}$, $\left.\mathrm{CDCl}_{3}\right) \delta 4.21\left(\mathrm{dd}, J=2.0,4.5 \mathrm{~Hz}, 2 \mathrm{H}, \mathrm{C} 1-\mathrm{H}_{2}\right), 4.29(\mathrm{dd}, J$ $=3.9,8.8 \mathrm{~Hz}, 1 \mathrm{H}$, one of $\left.\mathrm{CH}_{2} \mathrm{O}\right), 4.57\left(\mathrm{~s}, 2 \mathrm{H}, \mathrm{PhCH}_{2} \mathrm{O}\right)$, $4.71\left(\mathrm{t}, J=8.8 \mathrm{~Hz}, 1 \mathrm{H}\right.$, one of $\left.\mathrm{CH}_{2} \mathrm{O}\right), 5.49(\mathrm{dd}, J=3.9,8.8$ $\mathrm{Hz}, 1 \mathrm{H}, \mathrm{PhCHN}), 7.08$ (dt, $J=15.4,4.5 \mathrm{~Hz}, 1 \mathrm{H}, \mathrm{C} 2-H)$, 7.27-7.40 (m, 10H, ArH), $7.54(\mathrm{dt}, J=15.4,2.0 \mathrm{~Hz}, 1 \mathrm{H}$, $\mathrm{C} 3-H) ;{ }^{13} \mathrm{C}$ NMR $\left(67.8 \mathrm{MHz}, \mathrm{CDCl}_{3}\right) \delta 56.7(\mathrm{CH}), 68.8$ $\left(\mathrm{CH}_{2}\right), 69.8\left(\mathrm{CH}_{2}\right), 72.6\left(\mathrm{CH}_{2}\right), 120.2(\mathrm{CH}), 125.8(\mathrm{CH})$, $127.58(\mathrm{CH}), 127.63(\mathrm{CH}), 128.3(\mathrm{CH}), 128.5(\mathrm{CH}), 129.0$ $(\mathrm{CH}), 137.6(\mathrm{C}), 138.8(\mathrm{C}), 146.4(\mathrm{CH}), 153.4(\mathrm{C}), 164.0$ (C); FAB-HRMS $m / z$ calcd for $\mathrm{C}_{20} \mathrm{H}_{20} \mathrm{NO}_{4}(\mathrm{M}+\mathrm{H})^{+}$ 338.1392, found 338.1400; Anal. calcd for $\mathrm{C}_{20} \mathrm{H}_{19} \mathrm{NO}_{4}$ : C, 71.20; H, 5.68; N, 4.15, found: C, 71.27; H, 5.61; N, 4.26.

\section{3. $[4 R, 3(3 R)]-3-(4-B e n z y l o x y-3-m e t h y l b u t a n o y l)-4-$} phenyl-2-oxazolidinone (10). $\mathrm{MeMgBr}$ in $\mathrm{THF}$ (0.93 M, $127 \mathrm{~mL}, 119 \mathrm{mmol}$ ) was added to a suspension of $\mathrm{CuBr} \cdot \mathrm{SMe}_{2}(32.5 \mathrm{~g}, 158 \mathrm{mmol})$ in $3: 2 \mathrm{THF} / \mathrm{Me}_{2} \mathrm{~S}(400 \mathrm{~mL})$ at $-78{ }^{\circ} \mathrm{C}$ under an argon atmosphere. After stirring at $-78{ }^{\circ} \mathrm{C}$ for $20 \mathrm{~min}$ and at $0{ }^{\circ} \mathrm{C}$ for $20 \mathrm{~min}$, the yellow suspension was cooled to $-78{ }^{\circ} \mathrm{C}$, and a solution of carboximide 9 (13.3 g, $39.4 \mathrm{mmol})$ in $6: 1 \mathrm{THF} / \mathrm{CH}_{2} \mathrm{Cl}_{2}(140$ $\mathrm{mL}$ ) was added. After stirring at $-78{ }^{\circ} \mathrm{C}$ for $30 \mathrm{~min}$, the mixture was warmed to $-30{ }^{\circ} \mathrm{C}$ over $30 \mathrm{~min}$ and stirred at this temperature for $30 \mathrm{~min}$. The reaction was quenched with saturated aqueous $\mathrm{NH}_{4} \mathrm{Cl}(150 \mathrm{~mL})$, and the mixture was extracted with AcOEt $(300 \mathrm{~mL})$. The organic extract was successively washed with saturated aqueous $\mathrm{NH}_{4} \mathrm{Cl}$ $(2 \times 150 \mathrm{~mL}), \mathrm{H}_{2} \mathrm{O}(80 \mathrm{~mL})$ and brine $(2 \times 80 \mathrm{~mL})$, and dried over anhydrous $\mathrm{Na}_{2} \mathrm{SO}_{4}$. Filtration and evaporation in vacuo furnished the slightly yellow solid (14.4 g), whose ${ }^{1} \mathrm{H}$ NMR [integration of $\mathrm{C} 3-\mathrm{H}$, desired isomer 10 (2.68 $\mathrm{ppm})$, undesired isomer 11 (2.86 ppm)] revealed a 10/11 ratio of 94:6. Purification of the crude product by column chromatography (silica gel $300 \mathrm{~g}, 100: 1 \quad \mathrm{CH}_{2} \mathrm{Cl}_{2} / \mathrm{AcOEt}$ ) afforded a mixture of 1,4 -adducts $(13.6 \mathrm{~g}, 97 \%)$ as a white solid, which was recrystallized twice from $4: 1 \mathrm{AcOEt} / n$ hexane to afford desired isomer $10(7.06 \mathrm{~g}, 51 \%)$ as colorless needles. Concentration of the mother liquor followed by two recrystallizations from $4: 1 \mathrm{AcOEt} / n$ hexane gave $10(2.03 \mathrm{~g}, 15 \%)$ as colorless needles. This sequence was repeated again to provide another crop of carboximide $10(877 \mathrm{mg}, 6 \%)$, resulting in combined yield of $72 \%$ : TLC $R_{f}=0.23$ (4:1 $n$-hexane/AcOEt); mp 68.5$69.0{ }^{\circ} \mathrm{C} ;[\alpha]_{\mathrm{D}}{ }^{22}=-52.8\left(\mathrm{c} 2.08, \mathrm{CHCl}_{3}\right.$ ); IR (nujol) 2922 , 2855, 1782, 1699, 1462, 1381, 1300, 1200, 1121, 1036, 762, $729,698 \mathrm{~cm}^{-1} ;{ }^{1} \mathrm{H}$ NMR $\left(500 \mathrm{MHz}, \mathrm{CDCl}_{3}\right) \delta 0.94(\mathrm{~d}, J=$ $\left.6.8 \mathrm{~Hz}, 3 \mathrm{H}, \mathrm{C} 2-\mathrm{CH}_{3}\right), 2.42(\mathrm{~m}, 1 \mathrm{H}, \mathrm{C} 2-H), 2.68(\mathrm{dd}, J=6.2$, $16.1 \mathrm{~Hz}, 1 \mathrm{H}$, one of C3- $\left.\mathrm{H}_{2}\right), 3.25(\mathrm{dd}, J=7.3,16.1 \mathrm{~Hz}, 1 \mathrm{H}$, one of C3- $\left.H_{2}\right), 3.31\left(\mathrm{dd}, J=7.6,9.2 \mathrm{~Hz}, 1 \mathrm{H}\right.$, one of $\left.\mathrm{C} 1-H_{2}\right)$, $3.39\left(\mathrm{dd}, J=5.6,9.2 \mathrm{~Hz}, 1 \mathrm{H}\right.$, one of $\left.\mathrm{C} 1-H_{2}\right), 4.14(\mathrm{dd}, J=$ $3.8,8.8 \mathrm{~Hz}, 1 \mathrm{H}$, one of $\left.\mathrm{CH}_{2} \mathrm{O}\right), 4.36(\mathrm{t}, J=8.8 \mathrm{~Hz}, 1 \mathrm{H}$, one of $\left.\mathrm{CH}_{2} \mathrm{O}\right), 4.42\left(\mathrm{~d}, \mathrm{~J}=11.8 \mathrm{~Hz}, 1 \mathrm{H}\right.$, one of $\left.\mathrm{PhCH}_{2} \mathrm{O}\right), 4.44$ $\left(\mathrm{d}, J=11.8 \mathrm{~Hz}, 1 \mathrm{H}\right.$, one of $\left.\mathrm{PhCH}_{2} \mathrm{O}\right), 5.23(\mathrm{dd}, J=3.8,8.8$ $\mathrm{Hz}, 1 \mathrm{H}, \mathrm{PhCHN}), 7.23-7.37(\mathrm{~m}, 10 \mathrm{H}, \mathrm{Ar} H) ;{ }^{13} \mathrm{C} \mathrm{NMR}$ 
$\left(67.8 \mathrm{MHz}, \mathrm{CDCl}_{3}\right) \delta 17.1\left(\mathrm{CH}_{3}\right), 30.6(\mathrm{CH}), 39.2\left(\mathrm{CH}_{2}\right)$, $57.4(\mathrm{CH}), 69.5\left(\mathrm{CH}_{2}\right), 72.7\left(\mathrm{CH}_{2}\right), 75.0\left(\mathrm{CH}_{2}\right), 125.8(\mathrm{CH})$, $127.36(\mathrm{CH}), 127.43(\mathrm{CH}), 128.2(\mathrm{CH}), 128.4(\mathrm{CH}), 129.0$ (CH), 138.6 (C), 139.2 (C), 153.7 (C), 172.0 (C); FABHRMS $m / z$ calcd for $\mathrm{C}_{21} \mathrm{H}_{24} \mathrm{NO}_{4}(\mathrm{M}+\mathrm{H})^{+} 354.1705$, found 354.1718; Anal. calcd for $\mathrm{C}_{21} \mathrm{H}_{23} \mathrm{NO}_{4}$ : C, 71.37; $\mathrm{H}, 6.56$; , 3.96, found: $\mathrm{C}, 71.30 ; \mathrm{H}, 6.62 ; \mathrm{N}, 3.84$.

\section{4. $[4 S, 3(3 R)]-3-(4-B e n z y l o x y-3-m e t h y l b u t a n o y l)-4-$ phenyl-2-oxazolidinone (ent-11). Trimethylacetyl} chloride $(0.01 \mathrm{~mL}, 76.1 \mu \mathrm{mol})$ was added to a solution of $(R)$-4-benzyloxy-3-methylbutanoic acid ${ }^{22}(12.0 \mathrm{mg}, 57.6$ $\mu \mathrm{mol})$ and $\mathrm{Et}_{3} \mathrm{~N}(0.03 \mathrm{~mL}, 0.215 \mathrm{mmol})$ in THF $(1 \mathrm{~mL})$ at $-25^{\circ} \mathrm{C}$ under an argon atmosphere. After stirring at $-20{ }^{\circ} \mathrm{C}$ for $2 \mathrm{~h}, \mathrm{LiCl}(3.7 \mathrm{mg}, 87.3 \mu \mathrm{mol})$ and oxazolidinone ent-8 (9.5 mg, $58.2 \mu \mathrm{mol})$ were added, and the mixture was allowed to warm to room temperature. After stirring for $3 \mathrm{~h}$, the reaction was quenched with saturated aqueous $\mathrm{NaHCO}_{3}$ $(3 \mathrm{~mL})$, and the mixture was extracted with AcOEt $(15 \mathrm{~mL})$. The organic extract was successively washed with saturated aqueous $\mathrm{NaHCO}_{3}(2 \times 5 \mathrm{~mL})$ and brine $(2 \times 5 \mathrm{~mL})$, and dried over anhydrous $\mathrm{Na}_{2} \mathrm{SO}_{4}$. Filtration and evaporation in vacuo furnished the crude product $(21.7 \mathrm{mg}$, yellow oil), which was purified by column chromatography (silica gel 4 g, 4:1 $n$-hexane/AcOEt) to give ent-11 (19.1 mg, 94\%) as a colorless oil: TLC $R_{f}=0.23\left(4: 1 n\right.$-hexane/AcOEt); $[\alpha]_{\mathrm{D}}{ }^{18}$ $=+43.5$ (c 1.08, $\left.\mathrm{CHCl}_{3}\right)$; IR (film) 3032, 2961, 2927, 2857, $1781,1706,1455,1385,1325,1200,1121,1096,758,710$ $\mathrm{cm}^{-1} ;{ }^{1} \mathrm{H}$ NMR $\left(500 \mathrm{MHz}, \mathrm{CDCl}_{3}\right) \delta 0.94(\mathrm{~d}, J=6.9 \mathrm{~Hz}$, $\left.3 \mathrm{H}, \mathrm{C} 2-\mathrm{CH}_{3}\right), 2.37(\mathrm{~m}, 1 \mathrm{H}, \mathrm{C} 2-H), 2.86(\mathrm{dd}, J=8.0,16.6$ $\mathrm{Hz}, 1 \mathrm{H}$, one of C3- $\left.H_{2}\right), 3.04(\mathrm{dd}, J=5.7,16.6 \mathrm{~Hz}, 1 \mathrm{H}$, one of $\left.\mathrm{C} 3-\mathrm{H}_{2}\right), 3.30\left(\mathrm{dd}, J=6.9,9.2 \mathrm{~Hz}, 1 \mathrm{H}\right.$, one of $\left.\mathrm{C} 1-H_{2}\right)$, $3.35\left(\mathrm{dd}, J=5.7,9.2 \mathrm{~Hz}, 1 \mathrm{H}\right.$, one of $\left.\mathrm{C} 1-\mathrm{H}_{2}\right), 4.21(\mathrm{dd}, J=$ 3.4, 8.6 Hz, $1 \mathrm{H}$, one of $\left.\mathrm{CH}_{2} \mathrm{O}\right), 4.42\left(\mathrm{~s}, 2 \mathrm{H}, \mathrm{PhCH}_{2} \mathrm{O}\right), 4.56$ $\left(\mathrm{t}, J=8.6 \mathrm{~Hz}, 1 \mathrm{H}\right.$, one of $\left.\mathrm{CH}_{2} \mathrm{O}\right), 5.31(\mathrm{dd}, J=3.4,8.6 \mathrm{~Hz}$, $1 \mathrm{H}, \mathrm{PhCHN}), 7.20-7.40(\mathrm{~m}, 10 \mathrm{H}, \mathrm{ArH}) ;{ }^{13} \mathrm{C}$ NMR $(99.6$ $\left.\mathrm{MHz}, \mathrm{CDCl}_{3}\right) \delta 17.0\left(\mathrm{CH}_{3}\right), 30.2(\mathrm{CH}), 39.8\left(\mathrm{CH}_{2}\right), 57.6$ $(\mathrm{CH}), 69.9\left(\mathrm{CH}_{2}\right), 72.9\left(\mathrm{CH}_{2}\right), 74.8\left(\mathrm{CH}_{2}\right), 125.9(\mathrm{CH})$, $127.5(\mathrm{CH}), 127.6(\mathrm{CH}), 128.3(\mathrm{CH}), 128.6(\mathrm{CH}), 129.1$ (CH), 138.5 (C), 139.2 (C), 153.7 (C), 171.9 (C); FABHRMS $m / z$ calcd for $\mathrm{C}_{21} \mathrm{H}_{24} \mathrm{NO}_{4}(\mathrm{M}+\mathrm{H})^{+} 354.1705$, found 354.1715 .

\section{5. $[4 R, 3(2 R, 3 R)]-3-(4-B e n z y l o x y-2,3-d i m e t h y l b u t a n o-$} yl)-4-phenyl-2-oxazolidinone (12). A solution of carboximide 10 (14.1 g, $40.0 \mathrm{mmol})$ in THF (50 mL plus $2 \times 4 \mathrm{~mL}$ rinse) was added to a $0.5 \mathrm{M}$ solution of NaHMDS in THF (104 mL, $52.0 \mathrm{mmol})$ at $-78{ }^{\circ} \mathrm{C}$ under an argon atmosphere. After stirring for $30 \mathrm{~min}$, MeI $(12.5 \mathrm{~mL}, 199 \mathrm{mmol})$ was added, and the mixture was stirred for $6 \mathrm{~h}$. The reaction was quenched with saturated aqueous $\mathrm{NH}_{4} \mathrm{Cl}(50 \mathrm{~mL})$, and the mixture was extracted with AcOEt $(300 \mathrm{~mL})$. The organic extract was successively washed with saturated aqueous $\mathrm{NH}_{4} \mathrm{Cl}(2 \times 80 \mathrm{~mL})$ and brine $(100 \mathrm{~mL})$, and dried over anhydrous $\mathrm{Na}_{2} \mathrm{SO}_{4}$. Filtration and evaporation in vacuo furnished the crude product $(15.0 \mathrm{~g}$, yellow oil), whose diastereomeric ratio was determined to be $98: 2$ by ${ }^{1} \mathrm{H}-\mathrm{NMR}$ [integration of the benzylic proton at $\mathrm{C} 4$ of the oxazolidinone, desired syn-isomer 12 (4.91 ppm), undesired anti-isomer $(5.45 \mathrm{ppm})]$. Purification of the crude product by column chromatography (silica gel $300 \mathrm{~g}, 6: 1$ $\rightarrow$ 4:1 $\rightarrow$ 2:1 $n$-hexane/AcOEt) afforded syn-isomer 12 $(12.2 \mathrm{~g}, 83 \%)$ as a colorless oil, along with a $6.3: 1$ mixture of 10 and anti-isomer $(2.22 \mathrm{~g})$ as a white solid: TLC $R_{f}=$ 0.36 (4:1 n-hexane/AcOEt); $[\alpha]_{\mathrm{D}}{ }^{20}=-82.0$ (c 2.01, $\left.\mathrm{CHCl}_{3}\right)$; IR (film) 3032, 2969, 2932, 2878, 1779, 1705, 1454, 1383, 1319, 1204, 1098, 982, 741, $700 \mathrm{~cm}^{-1}$; ${ }^{1} \mathrm{H}$ NMR $(500 \mathrm{MHz}$, $\left.\mathrm{CDCl}_{3}\right) \delta 0.92\left(\mathrm{~d}, J=7.0 \mathrm{~Hz}, 3 \mathrm{H}, \mathrm{C} 2-\mathrm{CH}_{3}\right), 1.03(\mathrm{~d}, J=6.9$ $\left.\mathrm{Hz}, 3 \mathrm{H}, \mathrm{C} 3-\mathrm{CH}_{3}\right), 2.31(\mathrm{~m}, 1 \mathrm{H}, \mathrm{C} 2-H), 3.39$ (t, $J=9.3 \mathrm{~Hz}$, $1 \mathrm{H}$, one of $\left.\mathrm{C} 1-\mathrm{H}_{2}\right), 3.47(\mathrm{dd}, J=5.2,9.3 \mathrm{~Hz}, 1 \mathrm{H}$, one of $\left.\mathrm{C} 1-\mathrm{H}_{2}\right), 3.83(\mathrm{dq}, J=8.9,6.9 \mathrm{~Hz}, 1 \mathrm{H}, \mathrm{C} 3-H), 3.92-3.99(\mathrm{~m}$, $\left.2 \mathrm{H}, \mathrm{CH}_{2} \mathrm{O}\right), 4.39\left(\mathrm{~d}, J=11.4 \mathrm{~Hz}, 1 \mathrm{H}\right.$, one of $\left.\mathrm{PhCH}_{2} \mathrm{O}\right)$, $4.46\left(\mathrm{~d}, J=11.4 \mathrm{~Hz}, 1 \mathrm{H}\right.$, one of $\left.\mathrm{PhCH}_{2} \mathrm{O}\right), 4.91(\mathrm{dd}, J=4.7$, $8.4 \mathrm{~Hz}, 1 \mathrm{H}, \mathrm{PhCHN}), 7.15-7.18(\mathrm{~m}, 2 \mathrm{H}, \operatorname{Ar} H), 7.25-7.39$ $(\mathrm{m}, 8 \mathrm{H}, \mathrm{ArH}) ;{ }^{13} \mathrm{C} \mathrm{NMR}\left(67.8 \mathrm{MHz}, \mathrm{CDCl}_{3}\right) \delta 13.7\left(\mathrm{CH}_{3}\right)$, $14.9\left(\mathrm{CH}_{3}\right), 35.6(\mathrm{CH}), 40.0(\mathrm{CH}), 57.4(\mathrm{CH}), 69.1\left(\mathrm{CH}_{2}\right)$, $72.7\left(\mathrm{CH}_{2}\right), 75.4\left(\mathrm{CH}_{2}\right), 125.4(\mathrm{CH}), 127.4(\mathrm{CH}), 127.5$ $(\mathrm{CH}), 128.1(\mathrm{CH}), 128.2(\mathrm{CH}), 128.9(\mathrm{CH}), 138.6(\mathrm{C})$, 139.5 (C), 153.6 (C), 176.5 (C); FAB-HRMS $\mathrm{m} / \mathrm{z}$ calcd for $\mathrm{C}_{22} \mathrm{H}_{26} \mathrm{NO}_{4}(\mathrm{M}+\mathrm{H})^{+}$368.1862, found 368.1871; Anal. calcd for $\mathrm{C}_{22} \mathrm{H}_{25} \mathrm{NO}_{4}$ : C, 71.91; H, 6.86; N, 3.81, found: C, 71.99; $\mathrm{H}, 6.94 ; \mathrm{N}, 3.78$. Data for $[4 R, 3(2 S, 3 R)]$-isomer: TLC $R_{f}=$ $0.23\left(4: 1 n\right.$-hexane/AcOEt); $[\alpha]_{\mathrm{D}}{ }^{23}=-16.6\left(c 1.28, \mathrm{CHCl}_{3}\right)$; IR (nujol) 2924, 2854, 1786, 1701, 1457, 1377, 1319, 1211, $1083,749,714 \mathrm{~cm}^{-1}$; ${ }^{1} \mathrm{H}$ NMR $\left(500 \mathrm{MHz}, \mathrm{CDCl}_{3}\right) \delta 0.89(\mathrm{~d}$, $\left.J=6.9 \mathrm{~Hz}, 3 \mathrm{H}, \mathrm{C} 2-\mathrm{CH}_{3}\right), 1.08\left(\mathrm{~d}, J=6.9 \mathrm{~Hz}, 3 \mathrm{H}, \mathrm{C} 3-\mathrm{CH}_{3}\right)$, $2.16(\mathrm{~m}, 1 \mathrm{H}, \mathrm{C} 2-H), 3.14-3.24\left(\mathrm{~m}, 2 \mathrm{H}, \mathrm{C} 1-\mathrm{H}_{2}\right), 3.83$ (quintet, $J=6.9 \mathrm{~Hz}, 1 \mathrm{H}, \mathrm{C} 3-H), 4.22-4.33(\mathrm{~m}, 3 \mathrm{H}$, one of $\left.\mathrm{CH}_{2} \mathrm{O}, \mathrm{PhCH}_{2} \mathrm{O}\right), 4.68\left(\mathrm{t}, \mathrm{J}=9.2 \mathrm{~Hz}, 1 \mathrm{H}\right.$, one of $\left.\mathrm{CH}_{2} \mathrm{O}\right)$, $5.45(\mathrm{dd}, J=4.6,9.2 \mathrm{~Hz}, 1 \mathrm{H}, \mathrm{PhCHN}), 7.22-7.38(\mathrm{~m}, 10 \mathrm{H}$, $\mathrm{Ar} H) ;{ }^{13} \mathrm{C}$ NMR $\left(99.6 \mathrm{MHz}, \mathrm{CDCl}_{3}\right) \delta 13.0\left(\mathrm{CH}_{3}\right), 15.9$ $\left(\mathrm{CH}_{3}\right), 36.4(\mathrm{CH}), 40.5(\mathrm{CH}), 57.8(\mathrm{CH}), 69.5\left(\mathrm{CH}_{2}\right), 72.0$ $\left(\mathrm{CH}_{2}\right), 72.8\left(\mathrm{CH}_{2}\right), 126.2(\mathrm{CH}), 127.36(\mathrm{CH}), 127.39(\mathrm{CH})$, $128.3(\mathrm{CH}), 128.6(\mathrm{CH}), 129.0(\mathrm{CH}), 138.5(\mathrm{C}), 139.0(\mathrm{C})$, 153.4 (C), 176.1 (C); FAB-HRMS $m / z$ calcd for $\mathrm{C}_{22} \mathrm{H}_{26} \mathrm{NO}_{4}$ $(\mathrm{M}+\mathrm{H})^{+} 368.1862$, found 368.1848 .

4.6. (2R,3R)-4-Benzyloxy-2,3-dimethyl-1-butanol (16). A $2.0 \mathrm{M}$ solution of $\mathrm{LiBH}_{4}$ in THF $(21.5 \mathrm{~mL}, 43.0 \mathrm{mmol})$ was added to a solution of carboximide $12(12.7 \mathrm{~g}, 34.6$ $\mathrm{mmol})$ in THF $(120 \mathrm{~mL})$ and $\mathrm{H}_{2} \mathrm{O}(0.78 \mathrm{~mL}, 43.2 \mathrm{mmol})$ at $0{ }^{\circ} \mathrm{C}$. After stirring for $3 \mathrm{~h}$, the reaction mixture was allowed to warm to room temperature and stirred for $1.5 \mathrm{~h}$. The mixture was poured into an ice-cooled, two-layer mixture of $\mathrm{Et}_{2} \mathrm{O}(10 \mathrm{~mL})$ and saturated aqueous $\mathrm{NH}_{4} \mathrm{Cl}(60$ $\mathrm{mL}$ ), and the whole mixture was extracted with AcOEt $(150 \mathrm{~mL})$. The organic extract was successively washed with saturated aqueous $\mathrm{NH}_{4} \mathrm{Cl}(80 \mathrm{~mL})$ and brine $(2 \times 80$ $\mathrm{mL}$ ), and dried over anhydrous $\mathrm{Na}_{2} \mathrm{SO}_{4}$. Filtration and evaporation in vacuo furnished the crude product $(13.5 \mathrm{~g}$, white solid), which was purified by column chromatography (silica gel $250 \mathrm{~g}, 8: 1 n$-hexane/AcOEt $\rightarrow 4: 1 \mathrm{CH}_{2} \mathrm{Cl}_{2} /$ AcOEt) to give alcohol $16(5.22 \mathrm{~g}, 73 \%)$ as a colorless oil, along with recovered oxazolidinone $8(3.7 \mathrm{~g})$ as a white solid: TLC $R_{f}=0.31(3: 1 n$-hexane/AcOEt $) ;[\alpha]_{\mathrm{D}}^{21}=+6.19$ (c 2.00, $\mathrm{CHCl}_{3}$ ); IR (film) 3387 (br), 3030, 2876, 1454, 1364, 1094, 737, $698 \mathrm{~cm}^{-1} ;{ }^{1} \mathrm{H}$ NMR (500 MHz, $\left.\mathrm{CDCl}_{3}\right) \delta$ $0.90\left(\mathrm{~d}, J=7.0 \mathrm{~Hz}, 6 \mathrm{H}, \mathrm{C} 2-\mathrm{CH}_{3}, \mathrm{C} 3-\mathrm{CH}_{3}\right), 1.70(\mathrm{~m}, 1 \mathrm{H}$, C3-H), $1.87(\mathrm{~m}, 1 \mathrm{H}, \mathrm{C} 2-H), 2.20$ (br s, $1 \mathrm{H}, \mathrm{OH}), 3.38$ (dd, $J=7.2,9.3 \mathrm{~Hz}, 1 \mathrm{H}$, one of $\left.\mathrm{C} 1-H_{2}\right), 3.42(\mathrm{dd}, J=4.8,9.3$ $\mathrm{Hz}, 1 \mathrm{H}$, one of $\left.\mathrm{C} 1-\mathrm{H}_{2}\right), 3.48(\mathrm{dd}, J=5.8,11.1 \mathrm{~Hz}, 1 \mathrm{H}$, one of $\left.\mathrm{C} 4-\mathrm{H}_{2}\right), 3.60\left(\mathrm{dd}, J=5.0,11.1 \mathrm{~Hz}, 1 \mathrm{H}\right.$, one of C4- $\left.\mathrm{H}_{2}\right)$, 
$4.51\left(\mathrm{~d}, J=11.9 \mathrm{~Hz}, 1 \mathrm{H}\right.$, one of $\left.\mathrm{PhCH}_{2} \mathrm{O}\right), 4.53(\mathrm{~d}, J=11.9$ $\mathrm{Hz}, 1 \mathrm{H}$, one of $\left.\mathrm{PhCH}_{2} \mathrm{O}\right), 7.25-7.38(\mathrm{~m}, 5 \mathrm{H}, \mathrm{ArH}) ;{ }^{13} \mathrm{C}$ NMR $\left(67.8 \mathrm{MHz}, \mathrm{CDCl}_{3}\right) \delta 12.8\left(\mathrm{CH}_{3}\right), 13.1\left(\mathrm{CH}_{3}\right), 34.4$ $(\mathrm{CH}), 37.4(\mathrm{CH}), 65.7\left(\mathrm{CH}_{2}\right), 72.9\left(\mathrm{CH}_{2}\right), 73.9\left(\mathrm{CH}_{2}\right)$, $127.36(\mathrm{CH}), 127.42(\mathrm{CH}), 128.1(\mathrm{CH}), 138.0(\mathrm{C})$; EIHRMS $m / z$ calcd for $\mathrm{C}_{13} \mathrm{H}_{20} \mathrm{O}_{2}(\mathrm{M})^{+}$208.1463, found 208.1465; Anal. calcd for $\mathrm{C}_{13} \mathrm{H}_{20} \mathrm{O}_{2}$ : C, 74.96; $\mathrm{H}, 9.68$, found: C, 74.84; H, 9.71 .

4.7. (2R,3R)-4-Benzyloxy-2,3-dimethylbutanal (17). A solution of alcohol $16(2.77 \mathrm{~g}, 13.3 \mathrm{mmol})$ in $\mathrm{CH}_{2} \mathrm{Cl}_{2}(9$ $\mathrm{mL}$ ) was added to a solution of Dess-Martin periodinane $(6.80 \mathrm{~g}, 16.0 \mathrm{mmol})$ in $\mathrm{CH}_{2} \mathrm{Cl}_{2}(40 \mathrm{~mL})$ at $0{ }^{\circ} \mathrm{C}$ under an argon atmosphere. After stirring at room temperature for 10 min, the reaction was quenched with a mixture of $1 \mathrm{M}$ aqueous $\mathrm{Na}_{2} \mathrm{~S}_{2} \mathrm{O}_{3}(100 \mathrm{~mL})$ and saturated aqueous $\mathrm{NaHCO}_{3}$ $(100 \mathrm{~mL})$, and the whole mixture was extracted with AcOEt $(600 \mathrm{~mL})$. The organic extract was successively washed with saturated aqueous $\mathrm{NaHCO}_{3}(4 \times 100 \mathrm{~mL})$ and brine $(100 \mathrm{~mL})$, and dried over anhydrous $\mathrm{Na}_{2} \mathrm{SO}_{4}$. Filtration and evaporation in vacuo furnished the crude product $(2.90 \mathrm{~g}$, colorless oil), which was purified by column chromatography (silica gel $40 \mathrm{~g}, 10: 1 n$-hexane/ AcOEt) to give aldehyde $17(2.57 \mathrm{~g}, 94 \%)$ as a colorless oil: TLC $R_{f}=0.32(10: 1 n$-hexane/AcOEt $) ;[\alpha]_{\mathrm{D}}{ }^{21}=-39.8$ (c 2.16, $\mathrm{CHCl}_{3}$ ); IR (film) 2967, 2876, 1723, 1454, 1364, $1100,737,698 \mathrm{~cm}^{-1}$; ${ }^{1} \mathrm{H}$ NMR $\left(500 \mathrm{MHz}, \mathrm{CDCl}_{3}\right) \delta 0.87(\mathrm{~d}$, $\left.J=6.9 \mathrm{~Hz}, 3 \mathrm{H}, \mathrm{C} 2-\mathrm{CH}_{3}\right), 1.00\left(\mathrm{~d}, J=7.0 \mathrm{~Hz}, 3 \mathrm{H}, \mathrm{C} 3-\mathrm{CH}_{3}\right)$, $2.34(\mathrm{~m}, 1 \mathrm{H}, \mathrm{C} 2-H), 2.53(\mathrm{~m}, 1 \mathrm{H}, \mathrm{C} 3-H), 3.31(\mathrm{dd}, J=8.2$, $9.3 \mathrm{~Hz}, 1 \mathrm{H}$, one of $\left.\mathrm{C} 1-\mathrm{H}_{2}\right), 3.43(\mathrm{dd}, J=5.2,9.3 \mathrm{~Hz}, 1 \mathrm{H}$, one of $\left.\mathrm{C} 1-\mathrm{H}_{2}\right), 4.48\left(\mathrm{~d}, \mathrm{~J}=12.1 \mathrm{~Hz}, 1 \mathrm{H}\right.$, one of $\left.\mathrm{PhCH}_{2} \mathrm{O}\right)$, $4.49\left(\mathrm{~d}, J=12.1 \mathrm{~Hz}, 1 \mathrm{H}\right.$, one of $\left.\mathrm{PhCH}_{2} \mathrm{O}\right), 7.25-7.38(\mathrm{~m}$, $5 \mathrm{H}, \mathrm{Ar} H), 9.65(\mathrm{~d}, J=1.7 \mathrm{~Hz}, 1 \mathrm{H}, \mathrm{C} 4-H) ;{ }^{13} \mathrm{C} \mathrm{NMR}(67.8$ $\left.\mathrm{MHz}, \mathrm{CDCl}_{3}\right) \delta 8.6\left(\mathrm{CH}_{3}\right), 12.8\left(\mathrm{CH}_{3}\right), 33.5(\mathrm{CH}), 48.1$ $(\mathrm{CH}), 72.8\left(\mathrm{CH}_{2}\right), 127.3(\mathrm{CH}), 128.1(\mathrm{CH}), 138.0(\mathrm{C})$, $204.5(\mathrm{CH})$; EI-HRMS $\mathrm{m} / \mathrm{z}$ calcd for $\mathrm{C}_{13} \mathrm{H}_{18} \mathrm{O}_{2}(\mathrm{M})^{+}$ 206.1307, found 206.1315; Anal. calcd for $\mathrm{C}_{13} \mathrm{H}_{18} \mathrm{O}_{2}$ : $\mathrm{C}$, 75.69; H, 8.80, found: C, 75.49; H, 8.79.

4.8. Ethyl (4S,5R)-6-benzyloxy-4,5-dimethyl-2-hexenoate (18). Triethyl phosphonoacetate $(3.8 \mathrm{~mL}, 18.6 \mathrm{mmol})$ was added to a suspension of ${ }^{t} \mathrm{BuOK}(2.08 \mathrm{~g}, 18.5 \mathrm{mmol})$ in THF $(50 \mathrm{~mL})$ at $0{ }^{\circ} \mathrm{C}$ under an argon atmosphere. After stirring at room temperature for $30 \mathrm{~min}$, the solution was cooled to $-78{ }^{\circ} \mathrm{C}$, and a solution of aldehyde 17 (2.54 g, $12.3 \mathrm{mmol})$ in THF $(8 \mathrm{~mL}$ plus $2 \times 1 \mathrm{~mL}$ rinse $)$ was added. After $30 \mathrm{~min}$, the reaction mixture was allowed to warm to $0{ }^{\circ} \mathrm{C}$ and stirred for $30 \mathrm{~min}$. The reaction was quenched with saturated aqueous $\mathrm{NH}_{4} \mathrm{Cl}(30 \mathrm{~mL})$, and the whole mixture was extracted with AcOEt $(150 \mathrm{~mL})$. The organic extract was successively washed with saturated aqueous $\mathrm{NH}_{4} \mathrm{Cl}(40 \mathrm{~mL})$ and brine $(40 \mathrm{~mL})$, and dried over anhydrous $\mathrm{Na}_{2} \mathrm{SO}_{4}$. Filtration and evaporation in vacuo furnished the crude product $(5.30 \mathrm{~g}$, slightly yellow oil), which was purified by column chromatography (silica gel $150 \mathrm{~g}, 20: 1 n$-hexane/AcOEt) to give $\alpha, \beta$-unsaturated esters (E)-18 (3.02 g, 89\%) and (Z)-18 (368 mg, 11\%) as colorless oils. Data for $(2 E, 4 S, 5 R)$-isomer $(E)$-18: TLC $R_{f}=$ $0.29\left(10: 1 \quad n\right.$-hexane/AcOEt); $[\alpha]_{\mathrm{D}}^{22}=-46.2$ (c 2.09, $\mathrm{CHCl}_{3}$ ); IR (film) 2969, 2876, 1719, 1651, 1454, 1368, $1265,1184,737,698 \mathrm{~cm}^{-1} ;{ }^{1} \mathrm{H}$ NMR $\left(500 \mathrm{MHz}, \mathrm{CDCl}_{3}\right) \delta$ $0.90\left(\mathrm{~d}, J=6.9 \mathrm{~Hz}, 3 \mathrm{H}, \mathrm{C} 2-\mathrm{CH}_{3}\right), 1.00(\mathrm{~d}, J=6.8 \mathrm{~Hz}, 3 \mathrm{H}$, C3-CH $\left.\mathrm{CH}_{3}\right), 1.29\left(\mathrm{t}, J=7.1 \mathrm{~Hz}, 3 \mathrm{H}, \mathrm{OCH}_{2} \mathrm{CH}_{3}\right), 1.89(\mathrm{~m}, 1 \mathrm{H}$, C2-H), $2.49(\mathrm{~m}, 1 \mathrm{H}, \mathrm{C} 3-H), 3.29$ (dd, $J=6.3,9.3 \mathrm{~Hz}, 1 \mathrm{H}$, one of C1- $\left.\mathrm{H}_{2}\right), 3.37\left(\mathrm{dd}, J=6.4,9.3 \mathrm{~Hz}, 1 \mathrm{H}\right.$, one of $\left.\mathrm{C} 1-\mathrm{H}_{2}\right)$, $4.19\left(\mathrm{q}, J=7.1 \mathrm{~Hz}, 2 \mathrm{H}, \mathrm{OCH}_{2} \mathrm{CH}_{3}\right), 4.48\left(\mathrm{~s}, 2 \mathrm{H}, \mathrm{PhCH}_{2} \mathrm{O}\right)$, $5.78(\mathrm{dd}, J=0.9,15.7 \mathrm{~Hz}, 1 \mathrm{H}, \mathrm{C} 5-H), 6.93(\mathrm{dd}, J=7.6$, $15.7 \mathrm{~Hz}, 1 \mathrm{H}, \mathrm{C} 4-H), 7.25-7.36(\mathrm{~m}, 5 \mathrm{H}, \mathrm{Ar} H) ;{ }^{13} \mathrm{C} \mathrm{NMR}$ $\left(100.6 \mathrm{MHz}, \mathrm{CDCl}_{3}\right) \delta 13.5\left(\mathrm{CH}_{3}\right), 14.2\left(\mathrm{CH}_{3}\right), 14.6\left(\mathrm{CH}_{3}\right)$, $37.6(\mathrm{CH}), 37.9(\mathrm{CH}), 60.0\left(\mathrm{CH}_{2}\right), 72.9\left(\mathrm{CH}_{2}\right), 73.3\left(\mathrm{CH}_{2}\right)$, $120.1(\mathrm{CH}), 127.31(\mathrm{CH}), 127.34(\mathrm{CH}), 128.1(\mathrm{CH}), 138.3$ (C), $153.3(\mathrm{CH}), 166.6(\mathrm{C})$; EI-HRMS $\mathrm{m} / \mathrm{z}$ calcd for $\mathrm{C}_{17} \mathrm{H}_{24} \mathrm{O}_{3}(\mathrm{M})^{+} 276.1725$, found 276.1741; Anal. calcd for $\mathrm{C}_{17} \mathrm{H}_{24} \mathrm{O}_{3}$ : C, 73.88; $\mathrm{H}, 8.75$, found: $\mathrm{C}, 73.78 ; \mathrm{H}, 8.68$. Data for $(2 Z, 4 S, 5 R)$-isomer $(Z)$-18: TLC $R_{f}=0.35(10: 1 n$ hexane/AcOEt); $[\alpha]_{\mathrm{D}}{ }^{24}=+22.6\left(c 1.99, \mathrm{CHCl}_{3}\right)$; IR (film) 2976, 2874, 1721, 1644, 1454, 1416, 1182, 1098, 1032, 737, $698 \mathrm{~cm}^{-1} ;{ }^{1} \mathrm{H}$ NMR $\left(500 \mathrm{MHz}, \mathrm{CDCl}_{3}\right) \delta 0.91(\mathrm{~d}, J=6.3$ $\left.\mathrm{Hz}, 3 \mathrm{H}, \mathrm{C} 2-\mathrm{CH}_{3}\right), 1.00\left(\mathrm{~d}, J=6.3 \mathrm{~Hz}, 3 \mathrm{H}, \mathrm{C} 3-\mathrm{CH}_{3}\right), 1.27$ (t, $\left.J=7.1 \mathrm{~Hz}, 3 \mathrm{H}, \mathrm{OCH}_{2} \mathrm{CH}_{3}\right), 1.74(\mathrm{~m}, 1 \mathrm{H}, \mathrm{C} 2-H), 3.24(\mathrm{dd}$, $J=7.7,9.2 \mathrm{~Hz}, 1 \mathrm{H}$, one of $\left.\mathrm{C} 1-H_{2}\right), 3.43(\mathrm{dd}, J=5.0,9.2$ $\mathrm{Hz}, 1 \mathrm{H}$, one of $\left.\mathrm{C} 1-\mathrm{H}_{2}\right), 3.46(\mathrm{~m}, 1 \mathrm{H}, \mathrm{C} 3-H), 4.15(\mathrm{q}, J=$ $\left.7.1 \mathrm{~Hz}, 2 \mathrm{H}, \mathrm{OCH}_{2} \mathrm{CH}_{3}\right), 4.46(\mathrm{~d}, J=12.0 \mathrm{~Hz}, 1 \mathrm{H}$, one of $\left.\mathrm{PhCH}_{2} \mathrm{O}\right), 4.47\left(\mathrm{~d}, \mathrm{~J}=12.0 \mathrm{~Hz}, 1 \mathrm{H}\right.$, one of $\left.\mathrm{PhCH}_{2} \mathrm{O}\right), 5.71$ $(\mathrm{d}, J=11.5 \mathrm{~Hz}, 1 \mathrm{H}, \mathrm{C} 5-H), 6.09(\mathrm{t}, J=11.5 \mathrm{~Hz}, 1 \mathrm{H}, \mathrm{C} 4-H)$, 7.24-7.35 (m, 5H, ArH); ${ }^{13} \mathrm{C}$ NMR (100.6 MHz, $\left.\mathrm{CDCl}_{3}\right) \delta$ $14.2\left(\mathrm{CH}_{3}\right), 14.5\left(\mathrm{CH}_{3}\right), 17.1\left(\mathrm{CH}_{3}\right), 35.1(\mathrm{CH}), 38.6(\mathrm{CH})$, $59.7\left(\mathrm{CH}_{2}\right), 72.8\left(\mathrm{CH}_{2}\right), 74.1\left(\mathrm{CH}_{2}\right), 118.3(\mathrm{CH}), 127.2$ $(\mathrm{CH}), 127.3(\mathrm{CH}), 128.1(\mathrm{CH}), 138.5(\mathrm{C}), 154.4(\mathrm{CH})$, 166.1 (C); EI-HRMS $\mathrm{m} / \mathrm{z}$ calcd for $\mathrm{C}_{17} \mathrm{H}_{24} \mathrm{O}_{3}(\mathrm{M})^{+}$ 276.1725 , found 276.1713 .

\subsection{Ethyl (4S,5R)-6-hydroxy-4,5-dimethylhexanoate} (19). $\mathrm{Pd}(\mathrm{OH})_{2}$ on carbon $(20 \%, 123 \mathrm{mg})$ was added to a solution of enoate 18 (3.29 g, $11.9 \mathrm{mmol})$ in AcOEt (30 $\mathrm{mL}$ ) under an argon atmosphere, and the mixture was vigorously stirred for $5 \mathrm{~h}$ under 1 atm of hydrogen. The catalyst was filtered through a Celite pad, and the filtrate was evaporated in vacuo. Purification of the crude product (2.29 g, slightly yellow oil) by column chromatography (silica gel $65 \mathrm{~g}, 4: 1 n$-hexane/AcOEt) afforded hydroxyester $19(2.19 \mathrm{~g}, 97 \%)$ as a colorless oil: TLC $R_{f}=0.13(4: 1$ $n$-hexane/AcOEt); $[\alpha]_{\mathrm{D}}{ }^{22}=-10.6\left(c 2.40, \mathrm{CHCl}_{3}\right)$; IR (film) 3428 (br), 2961, 2928, 1736, 1181, $1036 \mathrm{~cm}^{-1}$; ${ }^{1} \mathrm{H}$ NMR $\left(500 \mathrm{MHz}, \mathrm{CDCl}_{3}\right) \delta 0.82\left(\mathrm{~d}, J=6.7 \mathrm{~Hz}, 3 \mathrm{H}, \mathrm{C} 3-\mathrm{CH}_{3}\right)$, $0.84\left(\mathrm{~d}, J=6.8 \mathrm{~Hz}, 3 \mathrm{H}, \mathrm{C} 2-\mathrm{CH}_{3}\right), 1.26(\mathrm{t}, J=7.2 \mathrm{~Hz}, 3 \mathrm{H}$, $\left.\mathrm{OCH}_{2} \mathrm{CH}_{3}\right), 1.50\left(\mathrm{~m}, 1 \mathrm{H}\right.$, one of $\left.\mathrm{C} 4-\mathrm{H}_{2}\right), 1.55-1.75(\mathrm{~m}, 3 \mathrm{H}$, $\mathrm{C} 2-\mathrm{H}, \mathrm{C} 3-\mathrm{H}$, one of $\left.\mathrm{C} 4-\mathrm{H}_{2}\right), 2.24-2.40\left(\mathrm{~m}, 2 \mathrm{H}, \mathrm{C} 5-\mathrm{H}_{2}\right)$, 3.48-3.57 (m, 2H, C1- $\left.H_{2}\right), 4.13(\mathrm{q}, J=7.2 \mathrm{~Hz}, 2 \mathrm{H}$, $\left.\mathrm{OCH}_{2} \mathrm{CH}_{3}\right) ;{ }^{13} \mathrm{C} \mathrm{NMR}\left(67.8 \mathrm{MHz}, \mathrm{CDCl}_{3}\right) \delta 11.3\left(\mathrm{CH}_{3}\right)$, $13.9\left(\mathrm{CH}_{3}\right), 14.0\left(\mathrm{CH}_{3}\right), 29.7\left(\mathrm{CH}_{2}\right), 32.3\left(\mathrm{CH}_{2}\right), 32.7(\mathrm{CH})$, $39.2(\mathrm{CH}), 60.1\left(\mathrm{CH}_{2}\right), 66.0\left(\mathrm{CH}_{2}\right), 174.0(\mathrm{C})$; FAB-HRMS $\mathrm{m} / \mathrm{z}$ calcd for $\mathrm{C}_{10} \mathrm{H}_{21} \mathrm{O}_{3}(\mathrm{M}+\mathrm{H})^{+} 189.1491$, found 189.1504; Anal. calcd for $\mathrm{C}_{10} \mathrm{H}_{20} \mathrm{O}_{3}: \mathrm{C}, 63.80 ; \mathrm{H}, 10.71$, found: $\mathrm{C}$, 63.71; H, 10.71.

4.10. (5S,6R)-5,6-Dimethyloxepan-2-one (20). $\mathrm{KOH}$ $(85 \%, 2.18 \mathrm{~g}, 33.0 \mathrm{mmol})$ was added to a solution of hydroxyester $19(2.07 \mathrm{~g}, 11.0 \mathrm{mmol})$ in $\mathrm{MeOH}(20 \mathrm{~mL})$ at $0{ }^{\circ} \mathrm{C}$. After stirring at room temperature for $4 \mathrm{~h}$, the solvent was removed in vacuo, and the residue was partitioned between AcOEt $(150 \mathrm{~mL})$ and $2 \mathrm{M}$ aqueous $\mathrm{NaHSO}_{4}(35$ 
$\mathrm{mL})$. The organic extract was washed with brine $(30 \mathrm{~mL})$ and dried over anhydrous $\mathrm{Na}_{2} \mathrm{SO}_{4}$. Filtration and evaporation in vacuo furnished the crude product $(1.96 \mathrm{~g})$, which was used without further purification.

TsOH (41.9 mg, $0.22 \mathrm{mmol}$ ) was added to a solution of the crude hydroxycarboxylic acid 5 (1.96 g) in benzene (800 $\mathrm{mL}$ ), and the mixture was refluxed for $1 \mathrm{~h}$. After the solvent was removed by distillation at atmospheric pressure, the residue was partitioned between AcOEt $(300 \mathrm{~mL})$ and saturated aqueous $\mathrm{NaHCO}_{3}(60 \mathrm{~mL})$. The organic extract was successively washed with saturated aqueous $\mathrm{NaHCO}_{3}$ $(60 \mathrm{~mL})$ and brine $(80 \mathrm{~mL})$, and dried over anhydrous $\mathrm{Na}_{2} \mathrm{SO}_{4}$. Filtration and concentration by atmospheric fractional distillation furnished the crude product $(1.89 \mathrm{~g})$, which was purified by column chromatography (silica gel $40 \mathrm{~g}, 5: 2 n$-hexane/ $\left.\mathrm{Et}_{2} \mathrm{O}\right)$ to give lactone 20 (1.41 g, 90\%) as a white solid: TLC $R_{f}=0.28(3: 1 n$-hexane/AcOEt $) ; \mathrm{mp}$ 41.0-42.0 ${ }^{\circ} \mathrm{C} ;[\alpha]_{\mathrm{D}}{ }^{21}=-43.1\left(c\right.$ 2.02, $\mathrm{CHCl}_{3}$ ); IR (nujol) 2922, 1750, 1458, 1377, 1277, 1074, $903 \mathrm{~cm}^{-1}$; ${ }^{1} \mathrm{H}$ NMR $\left(500 \mathrm{MHz} \mathrm{CDCl}_{3}\right) \delta 0.98\left(\mathrm{~d}, J=6.9 \mathrm{~Hz}, 3 \mathrm{H}, \mathrm{C} 2-\mathrm{CH}_{3}\right)$, $1.04\left(\mathrm{~d}, J=6.2 \mathrm{~Hz}, 3 \mathrm{H}, \mathrm{C} 3-\mathrm{CH}_{3}\right), 1.38-1.51$ (m, 2H, C3-H, one of $\left.\mathrm{C} 4-\mathrm{H}_{2}\right), 1.57(\mathrm{~m}, 1 \mathrm{H}, \mathrm{C} 2-\mathrm{H}), 1.89(\mathrm{~m}, 1 \mathrm{H}$, one of C4- $\left.\mathrm{H}_{2}\right), 2.60-2.69\left(\mathrm{~m}, 2 \mathrm{H}, \mathrm{C} 5-\mathrm{H}_{2}\right), 4.03(\mathrm{dd}, J=8.4,12.9$ $\mathrm{Hz}, 1 \mathrm{H}$, one of $\left.\mathrm{C} 1-H_{2}\right), 4.07(\mathrm{dd}, J=2.1,12.9 \mathrm{~Hz}, 1 \mathrm{H}$, one of $\left.\mathrm{C} 1-\mathrm{H}_{2}\right) ;{ }^{13} \mathrm{C} \mathrm{NMR}\left(67.8 \mathrm{MHz}, \mathrm{CDCl}_{3}\right) \delta 16.5\left(\mathrm{CH}_{3}\right)$, $20.4\left(\mathrm{CH}_{3}\right), 30.2\left(\mathrm{CH}_{2}\right), 32.4\left(\mathrm{CH}_{2}\right), 39.8(\mathrm{CH}), 40.0(\mathrm{CH})$, $72.9\left(\mathrm{CH}_{2}\right), 175.8(\mathrm{C})$; EI-HRMS $m / z$ calcd for $\mathrm{C}_{8} \mathrm{H}_{14} \mathrm{O}_{2}$ $(M)^{+}$142.0994, found 142.0997; Anal. calcd for $\mathrm{C}_{8} \mathrm{H}_{14} \mathrm{O}_{2}$ : C, 67.58; H, 9.92, found: C, 67.68; H, 9.73.

\subsection{1. (5S,6R)-5,6-Dimethyl-3-(phenylthiomethyl)oxepan-} 2-one (21). BuLi in $n$-hexane $(1.56 \mathrm{M}, 11.6 \mathrm{~mL}, 18.1$ mmol) was added to a solution of ${ }^{i} \operatorname{Pr}_{2} \mathrm{NH}(2.6 \mathrm{~mL}, 18.6$ $\mathrm{mmol})$ in THF $(20 \mathrm{~mL})$ at $-30{ }^{\circ} \mathrm{C}$ under an argon atmosphere. After stirring for $30 \mathrm{~min}$, the solution was cooled to $-78^{\circ} \mathrm{C}$, and a solution of lactone 20 (12.8 g, 9.00 $\mathrm{mmol})$ in THF $(10 \mathrm{~mL})$ was added. After $1 \mathrm{~h}$, TMSCl $(2.3$ $\mathrm{mL}, 18.1 \mathrm{mmol}$ ) was added, and the resulting mixture was stirred for $30 \mathrm{~min}$. The reaction mixture was allowed to warm to room temperature and stirred at this temperature for $1 \mathrm{~h}$. After the volatile elements were removed in vacuo, the residue was suspended in pentane $(60 \mathrm{~mL})$ and filtered through a plug of Celite. Evaporation of the filtrate in vacuo furnished the crude product $(2.91 \mathrm{~g})$, which was used without further purification.

$\mathrm{ZnBr}_{2}(20.3 \mathrm{mg}, 90.1 \mu \mathrm{mol})$ was added to a solution of the crude silyl ketene acetal and $\alpha$-chlorothioanisole $(1.8 \mathrm{~mL}$, $13.4 \mathrm{mmol})$ in $\mathrm{CH}_{2} \mathrm{Cl}_{2}(30 \mathrm{~mL})$ under an argon atmosphere. After stirring for $12 \mathrm{~h}$, the volatile elements were removed in vacuo, and the residue (5.21 g, orange oil) was purified by column chromatography (silica gel $90 \mathrm{~g}, 8: 1 n$-hexane/ AcOEt) to give sulfide $21(1.37 \mathrm{~g}, 58 \%)$ as a colorless oil, along with recovered lactone $20(168 \mathrm{mg}, 13 \%)$ as a white solid: TLC $R_{f}=0.39(4: 1 n$-hexane/AcOEt $) ;[\alpha]_{\mathrm{D}}^{23}=-1.20$ (c 2.01, $\mathrm{CHCl}_{3}$ ); IR (film) 2963, 2930, 1732, 1584, 1480, 1381, 1262, 1179, 1154, 1094, 741, $693 \mathrm{~cm}^{-1}$; ${ }^{1} \mathrm{H}$ NMR $\left(500 \mathrm{MHz}, \mathrm{C}_{6} \mathrm{D}_{6}\right) \delta 0.34\left(\mathrm{~d}, J=6.9 \mathrm{~Hz}, 1.5 \mathrm{H}, \mathrm{C} 2-\mathrm{CH}_{3}\right)$, $0.55-0.65\left(\mathrm{~m}, 3 \mathrm{H}, \mathrm{C} 3-\mathrm{CH}_{3}\right), 0.73(\mathrm{~m}, 0.5 \mathrm{H}, \mathrm{C} 3-H), 0.77$ (d, $\left.J=6.9 \mathrm{~Hz}, 1.5 \mathrm{H}, \mathrm{C} 2-\mathrm{CH}_{3}\right), 0.84(\mathrm{~m}, 0.5 \mathrm{H}, \mathrm{C} 2-H), 0.93-$ $1.09\left(\mathrm{~m}, 1 \mathrm{H}, \mathrm{C} 2-H\right.$, one of $\left.\mathrm{C} 4-H_{2}\right), 1.27(\mathrm{~m}, 0.5 \mathrm{H}, \mathrm{C} 3-H)$, $1.41-1.53\left(\mathrm{~m}, 1 \mathrm{H}, \mathrm{C} 4-H_{2}\right), 1.79(\mathrm{dd}, J=3.4,14.3 \mathrm{~Hz}, 0.5 \mathrm{H}$, one of $\left.\mathrm{C} 4-\mathrm{H}_{2}\right), 2.50-2.60(\mathrm{~m}, 1 \mathrm{H}, \mathrm{C} 5-\mathrm{H}), 2.76-2.87(\mathrm{~m}, 1 \mathrm{H}$, one of $\mathrm{CH}_{2} \mathrm{SPh}$ ), 3.23 (dd, $J=9.7,13.2 \mathrm{~Hz}, 0.5 \mathrm{H}$, one of $\left.\mathrm{C} 1-\mathrm{H}_{2}\right), 3.28\left(\mathrm{dd}, J=3.4,13.2 \mathrm{~Hz}, 0.5 \mathrm{H}\right.$, one of $\left.\mathrm{C} 1-\mathrm{H}_{2}\right)$, $3.41\left(\mathrm{dd}, J=1.7,13.2 \mathrm{~Hz}, 0.5 \mathrm{H}\right.$, one of $\left.\mathrm{C} 1-H_{2}\right), 3.605(\mathrm{~d}, J$ $=13.2 \mathrm{~Hz}, 0.5 \mathrm{H}$, one of $\left.\mathrm{C} 1-H_{2}\right), 3.609(\mathrm{dd}, J=4.6,13.7 \mathrm{~Hz}$, $0.5 \mathrm{H}$, one of $\left.\mathrm{CH}_{2} \mathrm{SPh}\right), 3.65(\mathrm{dd}, J=4.6,13.7 \mathrm{~Hz}, 0.5 \mathrm{H}$, one of $\left.\mathrm{CH}_{2} \mathrm{SPh}\right), 6.95(\mathrm{t}, J=7.4 \mathrm{~Hz}, 1 \mathrm{H}, \mathrm{ArH}), 7.03(\mathrm{t}, J=$ $7.4 \mathrm{~Hz}, 1 \mathrm{H}, \operatorname{Ar} H), 7.04$ (t, $J=7.4 \mathrm{~Hz}, 1 \mathrm{H}, \operatorname{Ar} H), 7.28(\mathrm{~d}, J$ $=7.4 \mathrm{~Hz}, 1 \mathrm{H}, \operatorname{Ar} H), 7.31(\mathrm{~d}, J=7.4 \mathrm{~Hz}, 1 \mathrm{H}, \operatorname{Ar} H) ;{ }^{13} \mathrm{C}$ NMR (67.8 MHz, $\left.\mathrm{CDCl}_{3}\right) \delta 16.3\left(\mathrm{CH}_{3}\right), 16.4\left(\mathrm{CH}_{3}\right), 19.0$ $\left(\mathrm{CH}_{3}\right), 20.6\left(\mathrm{CH}_{3}\right), 30.8\left(\mathrm{CH}_{2}\right), 34.0(\mathrm{CH}), 36.0\left(2 \times \mathrm{CH}_{2}\right)$, $36.9(\mathrm{CH}), 37.3(\mathrm{CH}), 37.4\left(\mathrm{CH}_{2}\right), 39.7(\mathrm{CH}), 40.8(\mathrm{CH})$, $41.2(\mathrm{CH}), 67.8\left(\mathrm{CH}_{2}\right), 73.3\left(\mathrm{CH}_{2}\right), 126.27(\mathrm{CH}), 126.32$ $(\mathrm{CH}), 128.9(\mathrm{CH}), 129.5(\mathrm{CH}), 129.6(\mathrm{CH}), 135.5(\mathrm{C})$, 135.6 (C), 175.90 (C), 175.93 (C); EI-HRMS m/z calcd for $\mathrm{C}_{15} \mathrm{H}_{20} \mathrm{O}_{2} \mathrm{~S}(\mathrm{M})^{+}$264.1184, found 264.1177; Anal. calcd for $\mathrm{C}_{15} \mathrm{H}_{20} \mathrm{O}_{2} \mathrm{~S}$ : C, 68.14; H, 7.63; S, 12.13, found: $\mathrm{C}, 68.02 ; \mathrm{H}$, 7.55; S, 12.15 .

4.12. (5S,6R)-5,6-Dimethyl-3-methyleneoxepan-2-one (2). A solution of sodium periodate $(1.17 \mathrm{~g}, 5.47 \mathrm{mmol})$ in $\mathrm{H}_{2} \mathrm{O}$ $(4 \mathrm{~mL})$ was added to a solution of sulfide 21 (1.31 g, 4.96 $\mathrm{mmol}$ ) in $\mathrm{MeOH}(36 \mathrm{~mL})$. After stirring for $24 \mathrm{~h}$, the mixture was filtrated through a Celite pad, and the filtrate was evaporated in vacuo. The residue was partitioned between AcOEt $(50 \mathrm{~mL})$ and $\mathrm{H}_{2} \mathrm{O}(20 \mathrm{~mL})$, and the aqueous layer was extracted with AcOEt $(50 \mathrm{~mL})$. The combined organic extracts were washed with brine $(20 \mathrm{~mL})$ and dried over anhydrous $\mathrm{Na}_{2} \mathrm{SO}_{4}$. Filtration and evaporation in vacuo furnished the crude product $(1.50 \mathrm{~g})$, which was purified by column chromatography (silica gel $45 \mathrm{~g}, 1: 2 \mathrm{n}$-hexane/AcOEt) to give sulfoxide (1.23 g, 89\%) as a white wax.

A solution of the sulfoxide $(1.23 \mathrm{~g}, 4.39 \mathrm{mmol})$ in toluene $(10 \mathrm{~mL})$ was refluxed for $3 \mathrm{~h}$. The solvent was evaporated in vacuo, and the residue ( $1.22 \mathrm{~g}$ ) was purified by column chromatography (silica gel $35 \mathrm{~g}, 6: 1 n$-hexane/Et $2 \mathrm{O}$ ) to give $\alpha$-methylene lactone $\mathbf{2}(572 \mathrm{mg}, 84 \%)$ as a white solid: TLC $R_{f}=0.34$ (4:1 $n$-hexane/AcOEt); mp 30.0-31.0 ${ }^{\circ} \mathrm{C}$; $[\alpha]_{\mathrm{D}}{ }^{21}=-6.69\left(c 2.01, \mathrm{CHCl}_{3}\right)$; IR (nujol) 2963, 2930, 1732, $1462,1308,1181,1140,1032,934,802 \mathrm{~cm}^{-1} ;{ }^{1} \mathrm{H}$ NMR $\left(500 \mathrm{MHz}, \mathrm{CDCl}_{3}\right) \delta 1.02\left(\mathrm{~d}, J=6.9 \mathrm{~Hz}, 3 \mathrm{H}, \mathrm{C} 2-\mathrm{CH}_{3}\right)$, $1.04\left(\mathrm{~d}, J=6.4 \mathrm{~Hz}, 3 \mathrm{H}, \mathrm{C} 3-\mathrm{CH}_{3}\right), 1.46-1.62(\mathrm{~m}, 2 \mathrm{H}, \mathrm{C} 2-\mathrm{H}$, C3-H), $2.17\left(\mathrm{dd}, J=10.7,14.6 \mathrm{~Hz}, 1 \mathrm{H}\right.$, one of C4- $\left.H_{2}\right), 2.50$ $\left(\mathrm{dd}, J=3.2,14.6 \mathrm{~Hz}, 1 \mathrm{H}\right.$, one of $\left.\mathrm{C} 4-H_{2}\right), 3.94(\mathrm{dd}, J=6.7$, $12.6 \mathrm{~Hz}, 1 \mathrm{H}$, one of $\left.\mathrm{C} 1-H_{2}\right), 4.15(\mathrm{dd}, J=2.1,12.6 \mathrm{~Hz}, 1 \mathrm{H}$, one of $\left.\mathrm{C} 1-\mathrm{H}_{2}\right), 5.43\left(\mathrm{~s}, 1 \mathrm{H}\right.$, one of $\left.\mathrm{C} 5=\mathrm{CH}_{2}\right), 5.75(\mathrm{~s}, 1 \mathrm{H}$, one of $\left.\mathrm{C} 5=\mathrm{CH}_{2}\right) ;{ }^{13} \mathrm{C}$ NMR $\left(67.8 \mathrm{MHz}, \mathrm{CDCl}_{3}\right) \delta 16.2$ $\left(\mathrm{CH}_{3}\right), 19.9\left(\mathrm{CH}_{3}\right), 38.2\left(\mathrm{CH}_{2}\right), 38.2(\mathrm{CH}), 39.3(\mathrm{CH}), 72.4$ $\left(\mathrm{CH}_{2}\right), 123.5\left(\mathrm{CH}_{2}\right), 141.0(\mathrm{C}), 172.4(\mathrm{C})$; EI-HRMS $\mathrm{m} / \mathrm{z}$ calcd for $\mathrm{C}_{9} \mathrm{H}_{14} \mathrm{O}_{2}(\mathrm{M})^{+}$154.0994, found 154.0999; Anal. calcd for $\mathrm{C}_{9} \mathrm{H}_{14} \mathrm{O}_{2}$ : C, 70.10; H, 9.15, found: C, 69.92; H, 9.19 . 


\section{Acknowledgments}

This research was supported in part by a Grant-in-Aid for Scientific Research on Priority Areas (A) "Exploitation of Multi-Element Cyclic Molecules" and Priority Areas 18032002 from the Ministry of Education, Culture, Sports, Science and Technology (MEXT) and by the Akiyama Foundation. We thank Ms. H. Matsumoto, A. Maeda, S. Oka, M. Kiuchi, and Mr. T. Hirose of the Center for Instrumental Analysis, Hokkaido University for technical assistance in mass and elemental analyses.

\section{References}

1. (a) Uemura, D.; Chou, T.; Haino, T.; Nagatsu, A.; Fukuzawa, S.; Zheng, S. Z.; Chen, H. S. J. Am. Chem. Soc. 1995, 117, 1155-1156; (b) Chou, T.; Kamo, O.; Uemura, D. Tetrahedron Lett. 1996, 37, 4023-4026; (c) Chou, T.; Haino, T.; Kuramoto, M.; Uemura, D. Tetrahedron Lett. 1996, 37, 4027-4030; (d) Takada, N.; Umemura, N.; Suenaga, K.; Chou, T.; Nagatsu, A.; Haino, T.; Yamada, K.; Uemura, D. Tetrahedron Lett. 2001, 42, 3491-3494.

2. Takada, N.; Umemura, N.; Suenaga, K.; Uemura, D. Tetrahedron Lett. 2001, 42, 3495-3497.

3. For a review, see: Kita, M.; Uemura, D. Chem. Lett. 2005, 34, 454-459.

4. For a review, see: O'Connor, P. D.; Brimble, M. A. Nat. Prod. Rep. 2007, 24, 869-885.

5. (a) McCauley, J. A.; Nagasawa, K.; Lander, P. A.; Mischke, S. G.; Semones, M. A.; Kishi, Y. J. Am. Chem. Soc. 1998, 120, 7647-7648; (b) Nagasawa, K. J. Synth. Org. Chem. Jpn. 2000, $58,877-886$.

6. Matsuura, F.; Hao, J.; Reents, R.; Kishi, Y. Org. Lett. 2006, 8, $3327-3330$.

7. (a) Matsuura, F.; Peters, R.; Anada, M.; Harried, S. S.; Hao, J.; Kishi, Y. J. Am. Chem. Soc. 2006, 128, 7463-7465; (b) Hao, J.; Matsuura, F.; Kishi, Y.; Kita, M.; Uemura, D.; Asai, N.; Iwashita, T. J. Am. Chem. Soc. 2006, 128, 7742-7743.

8. Sakamoto, S.; Sakazaki, H.; Hagiwara, K.; Kamada, K.; Ishii, K.; Noda, T.; Inoue, M.; Hirama, M. Angew. Chem., Int. Ed. 2004, 43, 6505-6510. For synthetic studies on pinnatoxin A by the Hirama group, see: (a) Noda, T.; Ishiwata, A.; Uemura, S.; Sakamoto, S.; Hirama, M. Synlett 1998, 298-300; (b) Ishiwata, A.; Sakamoto, S.; Noda, T.; Hirama, M. Synlett 1999, 692-694; (c) Nitta, A.; Ishiwata, A.; Noda, T.; Hirama, M. Synlett 1999, 695-696; (d) Wang, J.; Sakamoto, S.; Kamada, K.; Nitta, A.; Noda, T.; Oguri, H.; Hirama, M. Synlett 2003, 891-893.

9. (a) Sugimoto, T.; Ishihara, J.; Murai, A. Tetrahedron Lett. 1997, 38, 7379-7382; (b) Ishihara, J.; Sugimoto, T.; Murai, A. Synlett 1998, 603-606; (c) Sugimoto, T.; Ishihara, J.; Murai, A. Synlett 1999, 541-544; (d) Ishihara, J.; Tojo, S.; Kamikawa, A.; Murai, A. Chem. Commun. 2001, 1392-1393; (e) Ishihara, J.; Horie, M.; Shimada, Y.; Tojo, S.; Murai, A. Synlett 2002, 403-406.

10. Suthers, B. D.; Jacobs, M. F.; Kitching, W. Tetrahedron Lett. 1998, 39, 2621-2624.

11. (a) Pelc, M. J.; Zakarian, A. Org. Lett. 2005, 7, 1629-1631; (b) Pelc, M. J.; Zakarian, A. Tetrahedron Lett. 2006, 47, 7519-7523; (c) Lu, C.-D.; Zakarian, A. Org. Lett. 2007, 9, 3161-3163; (d) Stivala, C. E.; Zakarian, A. Tetrahedron Lett. 2007, 48, 6845-6848.

12. (a) Nakamura, S.; Inagaki, J.; Sugimoto, T.; Kudo, M.; Nakajima, M.; Hashimoto, S. Org. Lett. 2001, 3, 4075-4078; (b) Nakamura, S.; Inagaki, J.; Kudo, M.; Sugimoto, T.; Obara, K.; Nakajima, M.; Hashimoto, S. Tetrahedron 2002, 58, 10353-10374; (c) Nakamura, S.; Inagaki, J.; Sugimoto, T.; Ura, Y.; Hashimoto, S. Tetrahedron 2002, 58, 10375-10386.

13. (a) Roush, W. R.; Essenfeld, A. P.; Warmus, J. S.; Brown, B. B. Tetrahedron Lett. 1989, 30, 7305-7308; (b) Roush, W. R.; Brown, B. B. Tetrahedron Lett. 1989, 30, 7309-7312; (c) Roush, W. R.; Brown, B. B. J. Org. Chem. 1992, 57, 33803387; (d) Roush, W. R.; Brown, B. B. J. Org. Chem. 1993, 58, 2151-2161; (e) Roush, W. R.; Sciotti, R. J. J. Am. Chem. Soc. 1994, 116, 6457-6458; (f) Roush, W. R.; Reilly, M. L.; Koyama, K.; Brown, B. B. J. Org. Chem. 1997, 62, 87088721; (g) Roush, W. R.; Sciotti, R. J. J. Am. Chem. Soc. 1998, 120, 7411-7419; (h) Qi, J.; Roush, W. R. Org. Lett. 2006, 8, 2795-2798.

14. For the use of $\alpha$-methylene lactones in Diels-Alder reactions by other groups, see: (a) Fotiadu, F.; Michel, F.; Buono, G. Tetrahedron Lett. 1990, 31, 4863-4866; (b) Takeda, K.; Imaoka, I.; Yoshii, E. Tetrahedron 1994, 50, 10839-10848.

15. Nicolás, E.; Russell, K. C.; Hruby, V. J. J. Org. Chem. 1993, $58,766-770$.

16. The two research groups of NCI and Pfizer reported stereoselective preparation of $\alpha, \beta$-syn-disubstituted carboximides by the 1,4-addition/alkylation sequence using 4phenyl-2-oxazolidinone as a chiral auxiliary. NCI group (1. $\mathrm{H}_{2} \mathrm{C}=\mathrm{CHMgBr}$, 2. $\mathrm{XCH}_{2} \mathrm{CO}_{2}{ }^{t} \mathrm{Bu}$ ): (a) Wei, C.-Q.; Gao, Y.; Lee, K.; Guo, R.; Li, B.; Zhang, M.; Yang, D.; Burke, T. R., Jr. J. Med. Chem. 2003, 46, 244-254; (b) Shi, Z.-D.; Wei, C.Q.; Lee, K.; Liu, H.; Zhang, M.; Araki, T.; Roberts, L. R.; Worthy, K. M.; Fisher, R. J.; Neel, B. G.; Kelley, J. A.; Yang, D.; Burke, T. R., Jr. J. Med. Chem. 2004, 47, 2166-2169; (c) Kang, S.-U.; Shi, Z.-D.; Worthy, K. M.; Bindu, L. K.; Dharmawardana, P. G.; Choyke, S. J.; Bottaro, D. P.; Fisher, R. J.; Burke, T. R., Jr. J. Med. Chem. 2005, 48, 3945-3948; Pfizer group (1. $\mathrm{MeMgCl}, 2 . \mathrm{BrCH}_{2} \mathrm{CO}_{2}{ }^{t} \mathrm{Bu}$ ): (d) Belliotti, T. R.; Capiris, T.; Ekhato, I. V.; Kinsora, J. J.; Field, M. J.; Heffner, T. G.; Meltzer, L. T.; Schwarz, J. B.; Taylor, C. P.; Thorpe, A. J.; Vartanian, M. G.; Wise, L. D.; Zhi-Su, T.; Weber, M. L.; Wustrow, D. J. J. Med. Chem. 2005, 48, 22942307.

17. (a) Evans, D. A.; Chapman, K. T.; Hung, D. T.; Kawaguchi, A. T. Angew. Chem., Int. Ed. Engl. 1987, 26, 1184-1186; (b) Evans, D. A.; Chapman, K. T.; Bisaha, J. J. Am. Chem. Soc. 1988, 110, 1238-1256.

18. Ito, M.; Clark, C. W.; Mortimore, M.; Goh, J. B.; Martin, S. F J. Am. Chem. Soc. 2001, 123, 8003-8010.

19. Solladié, G.; Hutt, J.; Fréchou, C. Tetrahedron Lett. 1987, 28 , 61-64.

20. Ho, G.-J.; Mathre, D. J. J. Org. Chem. 1995, 60, 2271-2273.

21. For full characterization of the undesired isomer 11, we prepared ent-11 from (R)-4-benzyloxy-3-methylbutanoic acid $^{22}$ and $(S)$-4-phenyl-2-oxazolidinone (ent-8) utilizing the Merck protocol. ${ }^{20}$

22. Zhou, X.; Zhu, G.-D.; Haver, D. V.; Vandewalle, M.; De Clercq, P. J.; Verstuyf, A.; Bouillon, R. J. Med. Chem. 1999, 42, 3539-3556.

23. Evans and co-workers reported that alkylation of sodium enolates $\left(-78^{\circ} \mathrm{C}\right)$ is superior to the analogous reactions of the corresponding lithium enolates $\left(0{ }^{\circ} \mathrm{C}\right)$ and that small alkyl halides such as MeI are less stereoselective than their more sterically demanding counterparts: Evans, D. A.; Ennis, M. D.; Mathre, D. J. J. Am. Chem. Soc. 1982, 104, 1737-1739.

24. It has been demonstrated that the stereocontrol provided by $\beta$ substituents could override the facial bias conferred by the chiral auxiliary in the oxazolidinone-based alkylation reaction: Fernández-Zertuche, M.; Robledo-Pérez, R.; Meza- 
Aviña, M. E.; Ordoñez-Palacios, M. Tetrahedron Lett. 2002, 43, 3777-3780.

25. McGarvey, G. J.; Williams, J. M. J. Am. Chem. Soc. 1985, 107, 1435-1437.

26. Penning, T. D.; Djuric', S. W.; Haack, R. A.; Kalish, V. J.; Miyashiro, J. M.; Rowell, B. W.; Yu, S. S. Synth. Commun. 1990, 20, 307-312.

27. (a) Dess, D. B.; Martin, J. C. J. Org. Chem. 1983, 48, 41554156; (b) Dess, D. B.; Martin, J. C. J. Am. Chem. Soc. 1991, $113,7277-7287$.
28. Paterson, I. Tetrahedron 1988, 44, 4207-4219.

29. After submission of this manuscript, a total synthesis of pinnatoxin A was published: Stivala, C. E.; Zakarian, A. J. Am. Chem. Soc. 2008, 130, 3774-3776.

30. House, H. O.; Chu, C.-Y.; Wilkins, J. M.; Umen, M. J. J. Org. Chem. 1975, 40, 1460-1469.

31. Ireland, R. E.; Liu, L. J. Org. Chem. 1993, 58, 2899. 


\section{Graphical Abstract}

To create your abstract, type over the instructions in the template box below.

Fonts or abstract dimensions should not be changed or altered.

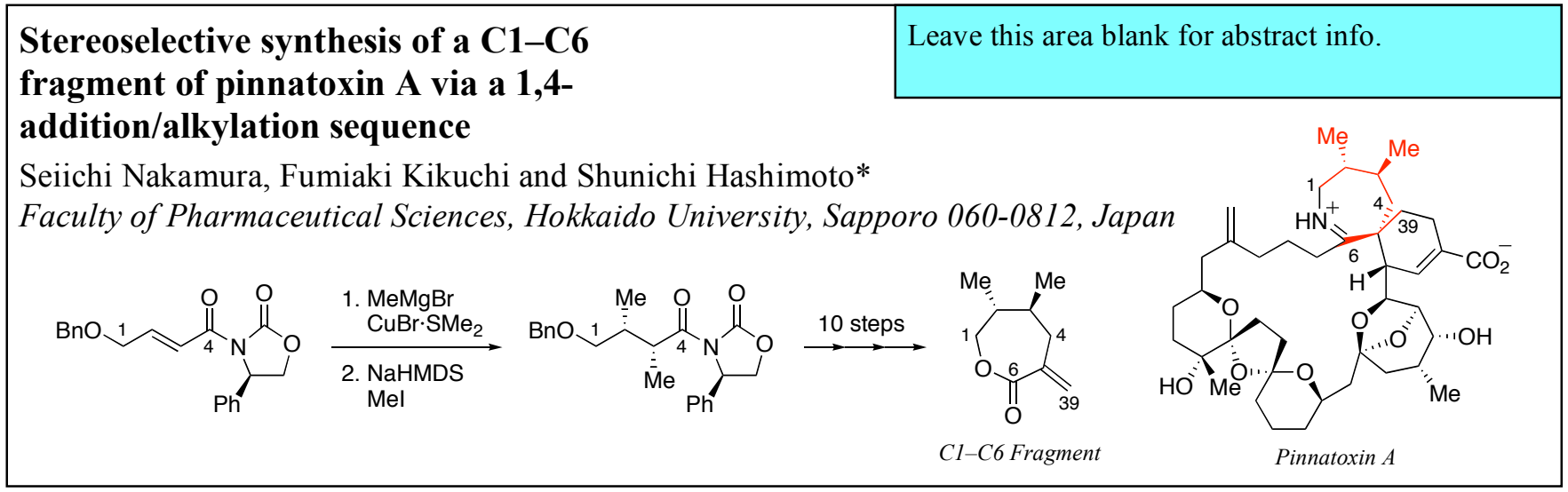




\section{Stereochemistry Abstract}

To create your abstract, type over the instructions in the template box below.

Fonts or abstract dimensions should not be changed or altered. You may insert more abstracts by copying this box or by using the menu option to insert a stereochemistry abstract.

Seiichi Nakamura, Fumiaki Kikuchi and Shunichi

Hashimoto*<smiles>O=C(/C=C/COc1ccccc1)N1C(=O)OCC1c1ccccc1</smiles>

$[\alpha]_{\mathrm{D}}^{26}=-67.3\left(c\right.$ 2.07, $\left.\mathrm{CHCl}_{3}\right)$

Source of chirality: D-phenylglycine

Absolute configuration: $(R)$

$\mathrm{C}_{20} \mathrm{H}_{19} \mathrm{NO}_{4}$

[4R,3(2E)]-3-(4-Benzyloxy-2-butenoyl)-4-phenyl-2-oxazolidinone

Seiichi Nakamura, Fumiaki Kikuchi and Shunichi

Hashimoto*

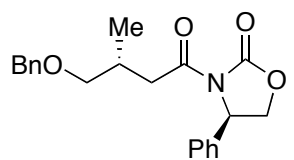

$[\alpha]_{\mathrm{D}}^{22}=-52.8\left(\right.$ c 2.08, $\left.\mathrm{CHCl}_{3}\right)$

Source of chirality: D-phenylglycine

Absolute configuration: $[4 R, 3(3 R)]$

$\mathrm{C}_{21} \mathrm{H}_{23} \mathrm{NO}_{4}$

[4R,3(3R)]-3-(4-Benzyloxy-3-methylbutanoyl)-4-phenyl-2-oxazolidinone

Seiichi Nakamura, Fumiaki Kikuchi and Shunichi

Hashimoto*

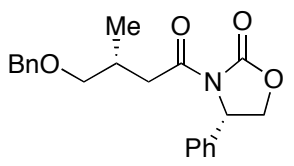

$[\alpha]_{\mathrm{D}}^{18}=+43.5\left(c 1.08, \mathrm{CHCl}_{3}\right)$

Source of chirality: D-phenylglycine

Absolute configuration: $[4 S, 3(3 R)]$

$\mathrm{C}_{21} \mathrm{H}_{23} \mathrm{NO}_{4}$

[4S,3(3R)]-3-(4-Benzyloxy-3-methylbutanoyl)-4-phenyl-2-oxazolidinone 
Seiichi Nakamura, Fumiaki Kikuchi and Shunichi

Hashimoto*<smiles>C[C@@H](COCc1ccccc1)C(=O)N1C(=O)OC[C@H]1c1ccccc1</smiles>

$\mathrm{C}_{22} \mathrm{H}_{25} \mathrm{NO}_{4}$
$[\alpha]_{\mathrm{D}}^{20}=-82.0\left(c 2.01, \mathrm{CHCl}_{3}\right)$

Source of chirality: D-phenylglycine

Absolute configuration: $[4 R, 3(2 R, 3 R)]$

[4R,3(2R,3R)]-3-(4-Benzyloxy-2,3-dimethylbutanoyl)-4-phenyl-2-oxazolidinone

Seiichi Nakamura, Fumiaki Kikuchi and Shunichi

Hashimoto*

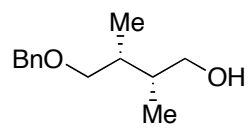

$\mathrm{C}_{13} \mathrm{H}_{20} \mathrm{O}_{2}$

$(2 R, 3 R)-4$-Benzyloxy-2,3-dimethyl-1-butanol
$[\alpha]_{\mathrm{D}}^{21}=+6.19\left(c 2.00, \mathrm{CHCl}_{3}\right)$

Source of chirality: D-phenylglycine

Absolute configuration: $(2 R, 3 R)$

Seiichi Nakamura, Fumiaki Kikuchi and Shunichi

Hashimoto*

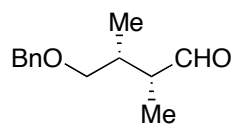

$\mathrm{C}_{13} \mathrm{H}_{18} \mathrm{O}_{2}$
$[\alpha]_{\mathrm{D}}^{21}=-39.8\left(\right.$ c $\left.2.16, \mathrm{CHCl}_{3}\right)$

Source of chirality: D-phenylglycine

Absolute configuration: $(2 R, 3 R)$

$(2 R, 3 R)-4-$ Benzyloxy-2,3-dimethylbutanal

Seiichi Nakamura, Fumiaki Kikuchi and Shunichi Hashimoto*<smiles>CCOC(=O)C=CC(C)C(C)COc1ccccc1</smiles>

$\mathrm{C}_{17} \mathrm{H}_{24} \mathrm{O}_{3}$
$[\alpha]_{\mathrm{D}}^{22}=-46.2\left(\right.$ c 2.09, $\left.\mathrm{CHCl}_{3}\right)$

Source of chirality: D-phenylglycine Absolute configuration: $(4 S, 5 R)$

Ethyl (2E,4S,5R)-6-benzyloxy-4,5-dimethyl-2-hexenoate 
Seiichi Nakamura, Fumiaki Kikuchi and Shunichi

Hashimoto*

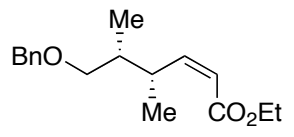

$\mathrm{C}_{17} \mathrm{H}_{24} \mathrm{O}_{3}$
$[\alpha]_{\mathrm{D}}^{24}=+22.6\left(c 1.99, \mathrm{CHCl}_{3}\right)$

Source of chirality: D-phenylglycine

Absolute configuration: $(4 S, 5 R)$

Ethyl (2Z,4S,5R)-6-benzyloxy-4,5-dimethyl-2-hexenoate

Seiichi Nakamura, Fumiaki Kikuchi and Shunichi

Hashimoto*<smiles>CCOC(=O)CCC(C)C(C)CO</smiles>

$\mathrm{C}_{10} \mathrm{H}_{20} \mathrm{O}_{3}$
$[\alpha]_{\mathrm{D}}^{22}=-10.6\left(\right.$ c $\left.2.40, \mathrm{CHCl}_{3}\right)$

Source of chirality: D-phenylglycine

Absolute configuration: $(4 S, 5 R)$

Ethyl (4S,5R)-6-hydroxy-4,5-dimethylhexanoate

Seiichi Nakamura, Fumiaki Kikuchi and Shunichi

Hashimoto*<smiles>CC1CCC(=O)OCC1C</smiles>

$[\alpha]_{\mathrm{D}}^{21}=-43.1\left(\right.$ c 2.02, $\left.\mathrm{CHCl}_{3}\right)$

Source of chirality: D-phenylglycine

Absolute configuration: $(5 S, 6 R)$

$\mathrm{C}_{8} \mathrm{H}_{14} \mathrm{O}_{2}$

(5S,6R)-5,6-Dimethyloxepan-2-one

Seiichi Nakamura, Fumiaki Kikuchi and Shunichi Hashimoto*<smiles>C=C1CC(C(C)C)COC1=O</smiles>

$[\alpha]_{D}^{21}=-6.69\left(c\right.$ 2.01, $\left.\mathrm{CHCl}_{3}\right)$

Source of chirality: D-phenylglycine

Absolute configuration: $(5 S, 6 R)$

$\mathrm{C}_{9} \mathrm{H}_{14} \mathrm{O}_{2}$

(5S,6R)-5,6-Dimethyl-3-methyleneoxepan-2-one 